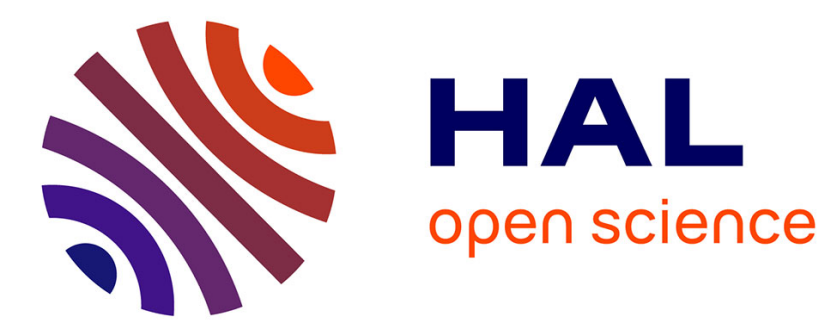

\title{
An infrared image processing to analyse the calorific effects accompanying strain localisation
}

\author{
André Chrysochoos, Hervé Louche
}

\section{To cite this version:}

André Chrysochoos, Hervé Louche. An infrared image processing to analyse the calorific effects accompanying strain localisation. International Journal of Engineering Science, 2000, 38 (16), pp.17591788. 10.1016/S0020-7225(00)00002-1 . hal-03349516

\section{HAL Id: hal-03349516 https://hal.science/hal-03349516}

Submitted on 20 Sep 2021

HAL is a multi-disciplinary open access archive for the deposit and dissemination of scientific research documents, whether they are published or not. The documents may come from teaching and research institutions in France or abroad, or from public or private research centers.
L'archive ouverte pluridisciplinaire HAL, est destinée au dépôt et à la diffusion de documents scientifiques de niveau recherche, publiés ou non, émanant des établissements d'enseignement et de recherche français ou étrangers, des laboratoires publics ou privés.

\section{다)(1) $(5$}

Distributed under a Creative Commons Attribution - NonCommercial| 4.0 International 


\title{
An infrared image processing to analyse the calorific effects accompanying strain localisation
}

\author{
André Chrysochoos *, Hervé Louche \\ Laboratoire de Mécanique et Génie Civil, Université Montpellier II, UMR 5508 CNRS, CC081, pl. E. Bataillon, 34095 \\ Montpellier Cedex, France
}

\begin{abstract}
This paper presents an infrared data processing developed to analyse the calorific manifestations accompanying elastoplastic transformation during tensile tests. The surface temperature images are provided by an experimental set-up essentially made of a testing machine coupled with an infrared camera equipped with a home-made numerizer. The 'inverse' passage from temperatures to heat sources is detailed in the case of flat and thin parallelepipedic samples. The infrared image processing, based on Fourier's techniques, was checked using spectral solutions of the heat equation in the case of realistic examples close to experiments. Numerical simulations are shown which attest coherence and efficiency of the method for several heat source distributions and different sets of noisy data. The method is then applied to experimental data files coming from tensile tests on mild steels at the room temperature. Sudden dissipative effects due to the propagation of the Lüders bands during the plastic plateau can be observed. Then, during the strain hardening, gradual and precocious concentrations of dissipation are shown; they herald the local necking of the sample. Finally, the interest of such experimental results is briefly discussed by referring to the specialised literature dealing with localisation phenomena and behaviour identification. (C) 2000 Elsevier Science Ltd. All rights reserved.
\end{abstract}

\section{Introduction}

The thermal and calorific effects associated with deformation processes have been observed for a long time by the experimenters. The first experiments in thermoelasticity were done by Lord Kelvin [1,2]. The pioneering calorimetric tests, performed by Taylor and Quinney [3], marked the

\footnotetext{
${ }^{*}$ Corresponding author.
} 
beginning of works on thermoplasticity. Of course, many authors have worked and continue to work today on the tricky problem of estimating, from temperature information, the heat evolved during materials deformation. Most of energy evaluations have been done by metallurgists. One must remember that by using energy balances, metallurgists like to draw up models capable of estimating the evolution of microstructural parameters such as dislocation density, dislocation cell size, etc. [4,5]. An important work of synthesis on this subject was published by Christian et al. [6]. However, the exploitation of energy data at the continuum scale is still nowadays relatively rare. For some, the use of a thermodynamic framework for constructing the behavioural constitutive equations is not yet a systematic attitude. For others, this 'phenomenological' approach of the materials behaviour is already judged old-fashioned, such authors generally prefer to develop multi-scales models within a purely mechanical framework.

However, we have to acknowledge that in the case of quasi-static tests, the temperature variations of solids often remain small and the theorist is naturally tempted to consider the deformation process as isotherm. This position, although natural from a strictly thermal viewpoint, at the same time deprives of any energy check of the behaviour modelling. Neglecting temperature variations induced by the deformation processes, it eliminates the possibility of establishing complete energy balances. Workers like Mandel and Bui had already underlined in [7] the great interest of combining mechanical and energy aspects of the same deformation phenomenon.

In this paper, we present an experimental set-up capable of storing the thermal images on the surface of thin and flat samples. These thermal images are used to estimate the heat source distributions by using the heat conduction equation. If a homogeneous mechanical test can reasonably be supposed, complete energy balances can be performed by linking the evolved heat estimations to the deformation energy deduced from the loading cell and from the extensometer signals (see [8-12]). In this paper, the thermal data are rather used to observe and analyse the calorific effects translating the localisation phenomena that occur during monotone and quasistatic tensile tests at constant room temperature.

In a first part, the thermomechanical framework used to interpret the experiment is reminded. The 'generalised standard material' formalism let us to introduce energy balance for thermoelastoplastic materials. After having briefly presented the main characteristics of the experimental arrangement, the infrared data processing is widely detailed and illustrated by several numerical examples developed to check its consistency. In a last part, experiments performed on mild steels are analysed. In particular, they show an inception of energy effects associated with the local necking a long time before the maximum force supported by the sample is reached.

\section{Thermomechanical framework}

Concepts and results of classical thermodynamics of irreversible processes (CTIP) are used $[13,14]$. At each instant $t$ of a quasi-static process, a thermodynamic equilibrium state of a homogeneous volume element is characterised by a set of $n+1$ state variables $\left(\alpha_{0}, \alpha_{1}, \ldots, \alpha_{n}\right)$.

As it is classically done in solid mechanics, we shall take as state variables: $T\left(T=\alpha_{0}\right)$, the absolute temperature, $\varepsilon\left(\varepsilon=\alpha_{1}\right)$, a strain tensor and $\alpha_{j}(j=2, \ldots, n)$, the components of a vector $\alpha$ of $n-1$ internal variables completing the description of the thermodynamic state. 
If $\psi$ and $s$ denote the specific Helmholtz free energy and the specific entropy, respectively, the Clausius-Duhem inequality that is derived from the local form of the second principle of thermodynamics, allows defining the dissipation $d$ as

$$
d=\boldsymbol{\sigma}: \mathbf{D}-\rho \psi_{, \varepsilon}: \dot{\boldsymbol{\varepsilon}}-\rho \psi_{, \alpha} \cdot \dot{\alpha}-\frac{\mathbf{q}}{T} \cdot \operatorname{grad} T \geqslant 0
$$

where $\boldsymbol{\sigma}$ is the Cauchy stress tensor, $\mathbf{D}$ the Eulerian strain rate tensor, $\rho$ the mass density, $\mathbf{q}$ the heat influx vector. The dot stands for the material time derivative. The thermodynamicists often introduce the irreversible entropy source defined by $\sigma_{\mathrm{s}}=d / T$. The equality $\sigma_{\mathrm{s}}=0$ (i.e. $\left.d=0\right)$ then characterises reversible thermodynamic processes. Classically, the intrinsic (mechanical) dissipation $d_{1}$ and the thermal dissipation $d_{2}$ are supposed to be separately positive and are respectively defined by

$$
d_{1}=\boldsymbol{\sigma}: \mathbf{D}-\rho \boldsymbol{\psi}_{, \varepsilon}: \dot{\boldsymbol{\varepsilon}}-\rho \boldsymbol{\psi}_{, \alpha} \cdot \dot{\alpha} \geqslant 0 \quad \text { and } \quad d_{2}=-\frac{\mathbf{q}}{T} \cdot \operatorname{grad} T \geqslant 0
$$

Per unit volume, the intrinsic dissipation $d_{1}$ is the difference between the anelastic energy rate $w_{\mathrm{a}}^{\prime}=\boldsymbol{\sigma}: \mathbf{D}-\rho \psi_{, \varepsilon}: \dot{\boldsymbol{\varepsilon}}$ and the stored energy rate $w_{\mathrm{s}}^{\prime}=\rho \psi_{, \alpha} \cdot \dot{\alpha}$.

Deduced from both principles of thermodynamics, the local heat conduction equation reads

$$
\rho C_{\varepsilon, \alpha} \dot{T}+\operatorname{div} \mathbf{q}=d_{1}+\rho T \boldsymbol{\psi}_{, \boldsymbol{T}, \varepsilon}: \dot{\boldsymbol{\varepsilon}}+\rho T \boldsymbol{\psi}_{, \boldsymbol{T}, \boldsymbol{\alpha}} \cdot \dot{\boldsymbol{\alpha}}+r_{\mathrm{e}},
$$

where $C_{\varepsilon, \alpha}$ denotes the specific heat capacity at $\varepsilon$ and $\alpha$ constant while $r_{\mathrm{e}}$ symbolises the external heat supply. The intrinsic dissipation $d_{1}$ and the thermomechanical coupling terms $\rho T \psi_{, \boldsymbol{T}, \varepsilon}: \dot{\boldsymbol{\varepsilon}}$ and $\rho T \boldsymbol{\psi}_{, \boldsymbol{T}, \boldsymbol{\alpha}} \cdot \dot{\boldsymbol{\alpha}}$ have been gathered in the right-hand member of (2.3). For metallic materials considered here, the 'Kelvin's' term $\rho T \psi_{, T, \varepsilon}: \dot{\boldsymbol{\varepsilon}}$ represents the volume heat rate due to thermoelastic couplings. Taking into account an isotropic conduction of heat $(\mathbf{q}=-k \operatorname{grad} T)$, we underline that the left-hand member of (2.3) can be interpreted as a partial derivative operator applied to the temperature.

\section{Experimental arrangement}

An original characteristic of the experimental set-up is the simultaneous recording of thermal and mechanical data: surface temperature, load applied to the sample, and elongation of its gauge part. This set-up (see Fig. 1) consists of a computerised uniaxial testing machine $(1,2)$ and an infrared thermography device $(4,5,6)$. This latter is made of an infrared camera (4), a display unit (5) and a home-made numerizer (6) allowing storage and processing of the thermal images in a second microcomputer (7).

The camera (Agema 880) is an infrared scanning system, with a single short wave detector InSb $([2-5.6] \mu \mathrm{m})$, liquid nitrogen cooled. The thermosignal in isothermal unity (IU), proportional to the thermal radiation, is digitised (DL), and then converted in temperature in Celcius degree $\left({ }^{\circ} \mathrm{C}\right)$ after a calibration operation. The numerisation system was initially conceived by [15], developed and improved by [16]. The temperature images are matrices of 256 lines per 180 columns digitised 


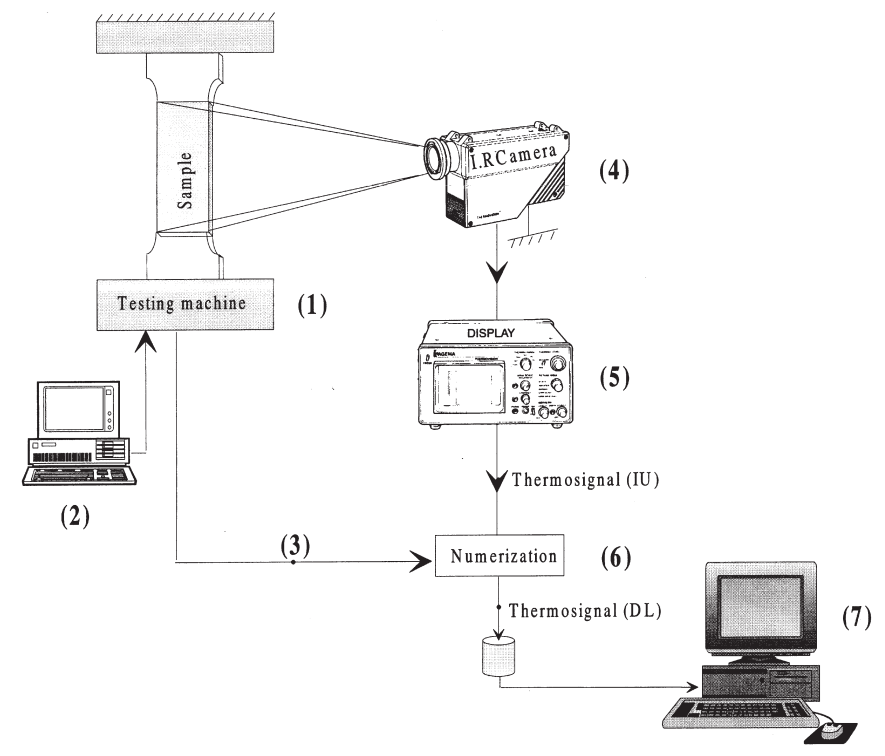

Fig. 1. Experimental set-up: (1) testing machine (DARTEC 9500), (2) control computer, (3) recording of global load and displacement, (4) infrared camera (AGEMA $880 \mathrm{SW}$ ), (5) display unit, (6) numerizer (12 bits), (7) storage and processing of thermal image.

on 12 bits. Moreover, the numerizer allows recording at the beginning of each line of a thermal image, by the means of (3), the corresponding load and elongation signals and four other electric signals like the thermal level and thermal range characterising the state of the camera.

\section{Toward simplified heat diffusion problems}

As mentioned above, the main object of the experimental data processing is to deduce from the surface temperature measurements the distribution of heat amounts evolved in the volume corresponding to the gauge length of the sample. For a real 3D medium, this operation, that belongs to the realm of 'inverse' problems, is tricky indeed, and even impossible to accomplish without any information on the source distributions to be quantified [17]. In what follows, the case, specific but important in practice, of thin and flat parallelepipedic samples will be considered. For such a 'simple' geometry, the heat sources and the temperatures are assumed to be homogeneously depth-wise distributed. On the sample surface, the sources will be locally determined by estimating a partial derivative operator applied to the temperature (the left-hand member of Eq. (2.3) being itself depth-wise averaged). Strictly speaking, this is no longer an inverse problem to solve insofar as the temperature field is, under such an assumption, completely known.

Moreover, in the case of the experimental conditions of our tests, the following hypotheses are done:

- the specific heat capacity $C_{\varepsilon, \alpha}$ and the isotropic conduction coefficient $k$ are material constants independent of the internal (hardening) state; 
- the external heat supply $r_{\mathrm{e}}$ is time independent;

- for quasi-static processes, the convective terms of the material time derivative are negligible;

- in the case of elastoplastic materials, couplings between temperature and hardening variables are neglected so that $\rho T \psi_{, \alpha, T} \cdot \dot{\alpha} \approx 0$.

These hypotheses are reasonable for many classical situations; they may become widely unsound when strong anisotropy pre-exists or develops during work-hardening, when first or second order phase transitions occur, or when the thermomechanical loading leads to dynamic instabilities.

\subsection{One-dimensional thermal diffusion problems}

For the sake of simplicity, a first data processing can be performed on a heat conduction equation averaged on a sample cross-section to get a 1D thermal diffusion problem (see Fig. 2(a)). Such an attitude can be compared to the one adopted in mechanics of curvilinear media. The following notations will be used as long as the gauge part of the sample remains a parallelepipedic structure: $\{e, l, L\}$ denotes the current geometrical parameters, $\left\{e_{0}, l_{0}, L_{0}\right\}$ being the initial depth, width, and length, respectively. We also note $\left\{x_{i}\right\}_{i=1,2,3}$ the Eulerian coordinates.

The averaged temperature variations $\bar{\theta}\left(x_{3}, t\right)$ and heat source $\bar{s}\left(x_{3}, t\right)$ for any cross-section $S=e \cdot l$ are then defined by:

$$
\begin{aligned}
& \bar{\theta}\left(x_{3}, t\right)=\frac{1}{S} \int_{0}^{e} \int_{-l / 2}^{l / 2}\left(T\left(x_{1}, x_{2}, x_{3}, t\right)-T_{0}\left(x_{1}, x_{2}, x_{3}, t\right)\right) \mathrm{d} x_{1} \mathrm{~d} x_{2}, \\
& \bar{s}\left(x_{3}, t\right)=\frac{1}{S} \int_{0}^{e} \int_{-l / 2}^{l / 2} w_{\mathrm{ch}}^{\prime} \mathrm{d} x_{1} \mathrm{~d} x_{2},
\end{aligned}
$$

where $T_{0}\left(x_{1}, x_{2}, x_{3}, t\right)$ is the (initial) equilibrium temperature field verifying $-k \Delta T_{0}=r_{\mathrm{e}}$, and where

$$
w_{\mathrm{ch}}^{\prime}=d_{1}+\rho T \frac{\partial^{2} \psi}{\partial T \partial \boldsymbol{\varepsilon}}: \dot{\boldsymbol{\varepsilon}}
$$
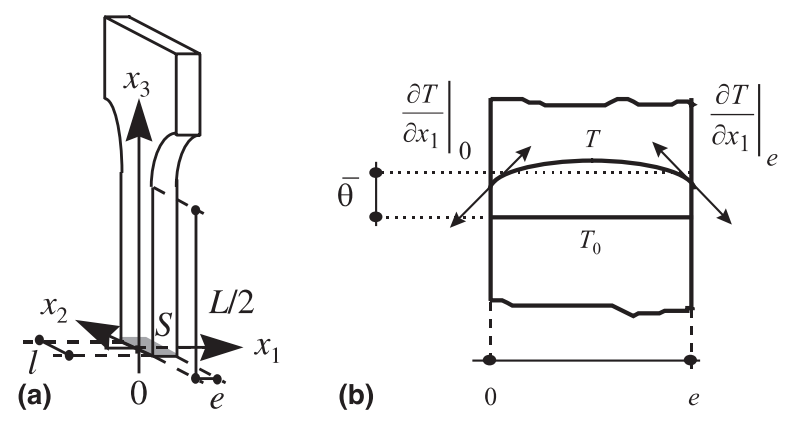

Fig. 2. (a) Basic sketch of an half sample; (b) illustration of the Fourier boundary conditions. 
Near thermal equilibrium, the thermal boundary conditions are well modelled by linear relations [18]. For the 'back' and 'front' boundary conditions we will suppose that

$$
\begin{aligned}
& \frac{\partial T\left(e, x_{2}, x_{3}, t\right)}{\partial x_{1}}=-\frac{\partial T\left(0, x_{2}, x_{3}, t\right)}{\partial x_{1}}, \\
& -k \frac{\partial T\left(e, x_{2}, x_{3}, t\right)}{\partial x_{1}}=\frac{h_{1}}{e} \int_{0}^{e}\left(T\left(x_{1}, x_{2}, x_{3}, t\right)-T_{0}\left(x_{1}, x_{2}, x_{3}, t\right)\right) \mathrm{d} x_{1} .
\end{aligned}
$$

The constant $h_{1}$ represents the lateral heat exchange coefficient between the surrounding air and the sample. The first equation assumes the symmetry of the thermal losses while the next one expresses the continuity of the heat flux. Assumption (4.4a) is reasonable as long as the heat sources are uniformly distributed along the little thickness of a homogeneous flat sample. In Eq. (4.4b), the losses by convection are assumed to be proportional to the mean thermal disequilibrium (see Fig. 2(b)), what is also fair as far as the temperature variations remain small.

To get, at all costs, a 1D analysis tool, the same kind of hypotheses can be adopted for the lateral boundaries $\left(x_{2}= \pm l / 2\right)$ :

$$
\begin{aligned}
& \frac{\partial T\left(x_{1}, l / 2, x_{3}, t\right)}{\partial x_{2}}=-\frac{\partial T\left(x_{1},-l / 2, x_{3}, t\right)}{\partial x_{2}}, \\
& -k \frac{\partial T\left(x_{1}, l / 2, x_{3}, t\right)}{\partial x_{2}}=\frac{h_{1}}{l} \int_{-l / 2}^{l / 2}\left(T\left(x_{1}, x_{2}, x_{3}, t\right)-T_{0}\left(x_{1}, x_{2}, x_{3}, t\right)\right) \mathrm{d} x_{2} .
\end{aligned}
$$

Naturally, in this $\mathrm{O} x_{2}$ direction, the validity of Eqs. (4.5a) and (4.5b) can be more easily at fault. But in the applications, it is of interest and often sufficient to do a simple analysis of thermoprofiles. The longitudinal boundary condition is written as

$$
-k \frac{\partial T\left(x_{1}, x_{2}, \pm L / 2, t\right)}{\partial x_{3}}= \pm h_{2}\left(T\left(x_{1}, x_{2}, \pm \frac{L}{2}, t\right)-T_{0}\left(x_{1}, x_{2}, \pm \frac{L}{2}, t\right)\right),
$$

where $h_{2}$ stands for the heat exchange coefficient between the grips of the testing machine and the sample. During the transformation, the exchange coefficients are assumed to be constant. The constant $\lambda_{2}^{-1}=k / h_{2}$ is a characteristic length of the longitudinal heat losses. The mean value on a cross-section of the material time derivative of the temperature can be deduced from the Leibniz rule and from the Gauss theorem in the case of an incompressible medium. In such a case, we get for any cross-section:

$$
\int_{0}^{e} \int_{-l / 2}^{l / 2} \frac{\mathrm{d}}{\mathrm{d} t} T\left(x_{1}, x_{2}, x_{3}, t\right) \mathrm{d} x_{1} \mathrm{~d} x_{2}=\frac{\mathrm{d}}{\mathrm{d} t} \int_{0}^{e} \int_{-l / 2}^{l / 2} T\left(x_{1}, x_{2}, x_{3}, t\right) \mathrm{d} x_{1} \mathrm{~d} x_{2} .
$$

With the use of this set of hypotheses and notations, a 1D differential problem can be expressed in the following form as soon as the convective terms of the material time derivative are neglected: 


$$
\begin{aligned}
& \rho C_{\varepsilon, \alpha} \frac{\partial \bar{\theta}\left(x_{3}, t\right)}{\partial t}+\frac{2 h_{1}(e+l)}{S} \bar{\theta}\left(x_{3}, t\right)-k \frac{\partial^{2} \bar{\theta}\left(x_{3}, t\right)}{\partial x_{3}^{2}}=\bar{s}\left(x_{3}, t\right), \\
& \bar{\theta}\left(x_{3}, 0\right)=0 \\
& \lambda_{2} \bar{\theta}\left( \pm \frac{L}{2}, t\right) \pm \partial \bar{\theta}\left( \pm \frac{L}{2}, t\right) / \partial x_{3}=0
\end{aligned}
$$

The most questionable hypothesis is certainly introduced in Eq. (4.5b). This relation implicitly admits that the mean width-wise temperature is a good representative of what happens along a line profile of the camera. This is obviously not always true. For instance, important lateral heat exchange characterised by very small $\lambda_{1}^{-1}$ (Dirichlet's condition) can lead to strong thermal gradient $\partial T / \partial x_{2}$ and invalidates Eq. (4.5b). In such cases, it is preferable to consider the following $2 \mathrm{D}$ approach.

\subsection{Two-dimensional thermal diffusion problems}

The extension to two dimensions thermal diffusion problems is simply realised averaging only depth-wise the temperature field. In order to lighten notations, we keep for this new averaging operation the same symbols as in Section 4.1. The averaged temperature variations $\bar{\theta}(\mathbf{x}, t)$ and heat source $\bar{s}(\mathbf{x}, t)$ at any point $\mathbf{x}=\left\{x_{2}, x_{3}\right\}$ on the gauge part of the sample surface are now defined by:

$$
\begin{aligned}
& \bar{\theta}(\mathbf{x}, t)=\frac{1}{e} \int_{0}^{e}\left(T\left(x_{1}, x_{2}, x_{3}, t\right)-T_{0}\left(x_{1}, x_{2}, x_{3}, t\right)\right) \mathrm{d} x_{1}, \\
& \bar{s}(\mathbf{x}, t)=\frac{1}{e} \int_{0}^{e} w_{\mathrm{ch}}^{\prime}\left(x_{1}, x_{2}, x_{3}, t\right) \mathrm{d} x_{1} .
\end{aligned}
$$

We also keep the 'back' and 'front' boundary conditions (4.4a), (4.4b) and the longitudinal boundary conditions (4.6) and the lateral boundary conditions becomes now

$$
-k \frac{\partial T\left(x_{1}, \pm l / 2, x_{3}, t\right)}{\partial x_{2}}= \pm h_{1}\left(T\left(x_{1}, \pm l / 2, x_{3}, t\right)-T_{0}\left(x_{1}, \pm l / 2, x_{3}, t\right)\right)
$$

By analogy with the 1D situation, the constant $\lambda_{1}^{-1}=k / h_{1}$ is the characteristic length of the lateral heat losses. The use of this new set of hypotheses leads us to define a $2 \mathrm{D}$ diffusion problem. It can be expressed in the following form when convective terms are neglected:

$$
\rho C_{\varepsilon, \alpha} \frac{\partial \bar{\theta}(\mathbf{x}, t)}{\partial t}+\frac{2 h_{1}}{e} \bar{\theta}(\mathbf{x}, t)-k \Delta \bar{\theta}(\mathbf{x}, t)=\bar{s}(\mathbf{x}, t)
$$




$$
\begin{aligned}
& \bar{\theta}(\mathbf{x}, 0)=0 \\
& \lambda_{1} \bar{\theta}\left( \pm \frac{l}{2}, x_{3}, t\right) \pm \partial \bar{\theta}\left( \pm \frac{l}{2}, x_{3}, t\right) / \partial x_{2}=0 \\
& \lambda_{2} \bar{\theta}\left(x_{2}, \pm \frac{L}{2}, t\right) \pm \partial \bar{\theta}\left(x_{2}, \pm \frac{L}{2}, t\right) / \partial x_{3}=0
\end{aligned}
$$

where $\Delta$ symbolises the 2D Laplacian operator.

\section{Infrared image processing}

The tricky goal of the thermal data processing is to deduce, from infrared images, the distributions of heat sources on the surface of the sample. Even if the temperature field is known, the passage to the mean heat sources $\bar{s}\left(x_{3}, t\right)$ or $\bar{s}(\mathbf{x}, t)$ is numerically strongly unstable because of the regularising effects of the heat diffusion mechanisms. The local heat sources evaluation is based on an estimate of the partial derivative operators constituting the left-hand member of Eqs. (4.8a) or (4.15a). This challenge is not obvious because thermal images are discrete, noisy, non-periodic and have a limited sampling rate. The image processing tools, used in what follows, are essentially founded on properties of the discrete Fourier transform (DFT) and on the properties of the Fourier series (FS). We will recall in what follows the conditions for which, the coefficients of the FS of a continuous signal are identical to those of the TFD of the corresponding sampled signal. This link between FS and TFD then allows the estimate of the derivative operators by using discrete data. The approximation of a FS by a TFD works only when the signal is periodic, bandlimited and without noise. In practice, operations of periodic extension and filtering will allow to tend towards this ideal situation. To lighten the presentation of the developed imaging techniques, we recall hereafter classical notations, definitions and main properties of the signals Fourier analysis.

\subsection{Brief reminder}

Let us consider an analog signal $h$ of one variable $u$, standing for time or space as soon as a map to map correspondence exists between time and space. Let $[0, a]$ be the record length. In such a situation, the signal is mathematically represented by a real function of real values. Generally, such a physical signal is termed 'finite energy' signal inasmuch as

$$
\int_{0}^{a}|h(u)|^{2} \mathrm{~d} u<+\infty
$$

and belongs, by definition, to the so-called functional space $L^{2}(0, a)$. 


\subsubsection{Fourier transform of signals}

A Fourier transform $H$ of the signal $h$ can be defined by extending its definition domain considering that $\tilde{h}(u)=h(u), \forall u \in[0, a]$, and $\tilde{h}(u)=0$ if $x<0$ or $x>a$, so that

$$
H(v)=\int_{-\infty}^{+\infty} \tilde{h}(u) \mathrm{e}^{-2 \mathrm{i} \pi v u} \mathrm{~d} u=\int_{0}^{a} h(u) \mathrm{e}^{-2 \mathrm{i} \pi v u} \mathrm{~d} u .
$$

In practice, it is very often impossible to work with analog (continuous) signals and the use of sampled data (digital signal) is inevitable. At this point, it is usual to interpret data as distributions so that if we consider now a regular sampling of $\tilde{h}$ using a sampling step $\tau_{\mathrm{e}}=a /(N-1)$ to get a number $N$ of data on $[0, a]$, the sampled 'function' $h_{\tau_{\mathrm{e}}}(u)$ can be written as:

$$
h_{\tau_{\mathrm{e}}}(u)=\sum_{n=-\infty}^{+\infty} \tilde{h}\left(n \tau_{\mathrm{e}}\right) \delta\left(u-n \tau_{\mathrm{e}}\right),
$$

where $\delta(u)$ is Dirac's distribution or the impulse function. The so-called 'Dirac's comb' is made of a sequence of equal distant impulse functions and plays a great role in signal processing inasmuch as Fourier transform of distributions can also be realised. Dirac's comb and its Fourier transform are defined by:

$$
\begin{gathered}
c_{\tau_{\mathrm{e}}}(u)=\sum_{n=-\infty}^{+\infty} \delta\left(u-n \tau_{\mathrm{e}}\right), \\
C_{v_{\mathrm{e}}}(v)=v_{\mathrm{e}} \sum_{n=-\infty}^{+\infty} \delta\left(v-n v_{\mathrm{e}}\right),
\end{gathered}
$$

where $v_{\mathrm{e}}=1 / \tau_{\mathrm{e}}$ is the sampling frequency. The sampling operation and the Fourier transform corresponding to the sampled signal can be formally rewritten as

$$
\begin{aligned}
& h_{\tau_{\mathrm{e}}}(u)=\tilde{h}(u) \times c_{\tau_{\mathrm{e}}}(u), \\
& H_{v_{\mathrm{e}}}(v)=H(v) * C_{v_{\mathrm{e}}}(v),
\end{aligned}
$$

where the star * symbolises the convolution product. Note that $H_{v_{\mathrm{e}}}$ is a continuous and periodic function with period $v_{\mathrm{e}}$. In accordance with these properties, it is possible to derive the definition of the DFT well adapted to digital machine computation. An inverse Fourier transform of the sampled spectrum, realised at the sampling rate $v_{a}=1 / a=v_{\mathrm{e}} / N$, leads to a periodic extension of the discrete motif associated to $h$ defined on $[0, a]$. Linear relationships can be established between $N$ values on a period of the sampled function $h_{n}=\left(h_{a}^{\tau_{\mathrm{e}}}\right)_{n}=h\left(n \tau_{\mathrm{e}}\right)$ and $N$ values extracted from a period in the frequency domain $H_{n}=\left(H_{v_{\mathrm{e}}}^{v_{a}}\right)_{n}=H_{v_{\mathrm{e}}}\left(n v_{a}\right)$. These are the famous relations defining the DFT and the discrete inverse Fourier transform (DIFT). They can be written as 


$$
\begin{aligned}
& h_{k}=\sum_{n=0}^{N-1} H_{n} \exp \left(2 \mathrm{i} \pi n \frac{k}{N}\right) \quad k=0,1, \ldots, N-1, \\
& H_{n}=\frac{1}{N} \sum_{k=0}^{N-1} h_{k} \exp \left(-2 \mathrm{i} \pi n \frac{k}{N}\right) \quad n=0,1, \ldots, N-1 .
\end{aligned}
$$

In what follows, we will note $\mathscr{F}_{d}$ and $\mathscr{F}_{d}^{-1}$ these both operations, respectively, so that we will formally write $H=\mathscr{F}_{d}(h)$ and $h=\mathscr{F}_{d}^{-1}(H)$.

\subsubsection{Fourier series and waveform sampling}

Another classical attitude for extending the continuous function $h$ is to consider its periodic extension, rippling its waveform motif. A new function $h_{a}$ with period $a$ is then defined by $h_{a}(u)=h(u), \forall u \in[0, a]$ and $h_{a}(u+n a)=h(u), \forall u \in[0, a[, n \in Z$. The periodicity property allows expressing $h_{a}$ as a Fourier series

$$
h_{a}(u)=\frac{p_{0}}{2}+\sum_{n=1}^{\infty}\left[p_{n} \cos \left(2 \pi n v_{a} u\right)+q_{n} \sin \left(2 \pi n v_{a} u\right)\right]
$$

where $v_{a}$ is the fundamental frequency equals to $1 / a$. The coefficients of the sinusoids $p_{n}$ and $q_{n}$ are classically given by the integrals

$$
\begin{aligned}
& p_{n}=\frac{2}{a} \int_{-a / 2}^{a / 2} h_{a}(u) \cos \left(2 \pi n v_{a} u\right) \mathrm{d} u, \quad n=0,1,2,3, \ldots, \\
& q_{n}=\frac{2}{a} \int_{-a / 2}^{a / 2} h_{a}(u) \sin \left(2 \pi n v_{a} u\right) \mathrm{d} u, \quad n=1,2,3, \ldots
\end{aligned}
$$

If $\alpha_{n}=1 / 2\left(p_{n}-\mathrm{i} q_{n}\right), n=0, \pm 1, \pm 2, \ldots$, denote the complex coefficients of the Fourier series, $h_{a}$ may be written in exponential form as

$$
h_{a}(u)=\sum_{n=-\infty}^{+\infty} \alpha_{n} \exp \left(2 \mathrm{i} \pi n \frac{u}{a}\right)
$$

Let us recall that an important relationship between the coefficients $H_{n}$ of the DFT and the coefficients $\alpha_{n}$ of the Fourier series can be established. For $0 \leqslant n<N$, we can write

$$
H_{n}-\alpha_{n}=\sum_{q \neq 0} \alpha_{n+q N}, \quad q \in Z
$$

Such a result shows that the more rapidly the coefficients of the Fourier series tend to zero, the better is the approximation $H_{n} \approx \alpha_{n}$ for $0 \leqslant n<N$. In other words, the more regular the function 
$h_{a}$ is, the more the sum $\left|\cdots+\alpha_{-2 N+n}+\alpha_{-N+n}+\alpha_{N+n}+\alpha_{2 N+n}+\cdots\right|$ is negligible and the better the DFT approximates a Fourier series [21].

For instance, if $h_{a}$ is a trigonometric polynomial, the discrete Fourier transform coefficients are equal to the ones of the Fourier series as soon as the number $N$ of data (related to the sampling rate) is twice as big as the degree of the polynomial. Conversely, if the periodic function $h_{a}$ presents discontinuities, the approximation can become bad. It is the case as soon as $h(0) \neq h(a)$, what happens quasi systematically with the thermal images.

\subsection{Image processing constraints}

\subsubsection{Sampling constraint}

In practice, the choice of the sampling rate $v_{\mathrm{e}}$ is of major importance. Upper limited by the digitizer (performances of the analog-digital converter, storage rate capacity, etc.), the sampling frequency must however be sufficiently high to avoid the famous aliasing effect. To illustrate the potentialities of Fourier analysis, let us recall that the sampling theorem (the Shannon theorem) states that if the spectrum of an analog signal $h$ is band-limited at the frequency $v_{\mathrm{c}}$ and if the sampling frequency verifies $v_{\mathrm{e}} \geqslant 2 v_{\mathrm{c}}$, then the analog signal $h(u)$ can be uniquely determined from a knowledge of its sampled values

$$
h(u)=\tau_{\mathrm{e}} \sum_{n=-\infty}^{n=+\infty} h_{n} \frac{\sin 2 \pi v_{\mathrm{c}}\left(u-n \tau_{\mathrm{e}}\right)}{\pi\left(u-n \tau_{\mathrm{e}}\right)} .
$$

Unfortunately, the presence of noise on the thermal data does not make it possible to exactly know if the thermal signal is band-limited. However, the use of a low-pass convolutive filter allows avoiding an aliasing effect even if it involves a possible vanishing of the high part of the thermal signal frequencies.

\subsubsection{Periodic extension of analog signals}

To get a development in the Fourier series of an analog signal that is time-limited, or spacelimited as well, a natural attitude is to consider its periodic extension. If $h_{a}$ is such that $h_{a}(0) \neq h_{a}(a)$, the rippling reveals sharp discontinuities that induce apparition of new frequency components in the Fourier series. These additional frequency components can be mixed with those of noise and make the filtering problem more complicated. This classical phenomenon is often termed leakage effect in the specialised literature [19]. To reduce this leakage, a regular extension of the signal $h$ from $a$ to $a^{\prime}>a$ can be planned to define a new periodic waveform $h_{a^{\prime}}$ such that $h_{a^{\prime}}\left(a^{\prime}\right)=h_{a^{\prime}}(0)$. Note that several extension on $\left[a, a^{\prime}\right]$ can be proposed like folding (mirror image) about ordinate axis, polynomial extensions, etc. The better the extension is, the more the signal is band-limited. Note also that zero-padding nearly always offered in signal processing toolboxes is not a very efficacious method to settle this leakage problem. Moreover in our case, the influence of the parasitic frequencies due to the non-periodicity of images is strongly amplified by the presence of the Laplacian operator during the heat sources evaluation. We shall see in Section 5.4 that a periodic extension of images were planned before evaluating the heat sources. 


\subsection{Thermal noise characteristics}

Digitised thermal data are always noisy. This noise has several origins: it comes from the infrared detector itself (InSb detector, liquid nitrogen cooled), from the digitizer (electronic components, sampling and quantization of data) and finally from the parasitic reflections on the surface of the observed object. A global study of the temperature noise was performed to calculate the thermal resolution (the noise equivalent temperature difference - NETD) and the noise characteristics (mean value, standard deviation, ...). Both are useful to guide in the choice of an image processing method. To extract the noise, images were recorded during a stationary thermal scene (camera lens sealed by a mask, a black body in thermal equilibrium with surrounding).

Examples of noise profiles are presented in Fig. 3(a) and (b). The data analysis shows that the thermosignal is disrupted by a white noise characterised by a Gaussian probability distribution of mean 0 (Fig. 3(c)). Its power spectrum is approximately uniform (Fig. 3(d)). This last result unfortunately implies that the numerical filter, whatever its characteristics, is unable to eliminate completely the parasitic frequencies. That is the reason why the efficiency of the data processing was checked by using numerical tests close to experiments.

\subsection{Heat sources evaluation}

To estimate the distribution of heat sources on the surface of the specimen, we first use a parabolic extension of images. The first and the second order space derivatives of the temperature are estimated along the image sides by using least-square approximations. For the 1D analysis, the extension is such that the extended profile has $2^{n}$ points $(n \in N)$ to be allowed to use the fast Fourier transform (FFT) algorithms. Typically, $n$ is about 8 . In the case of images analysis, we arbitrarily decided to take an extension size corresponding with the image width. The data
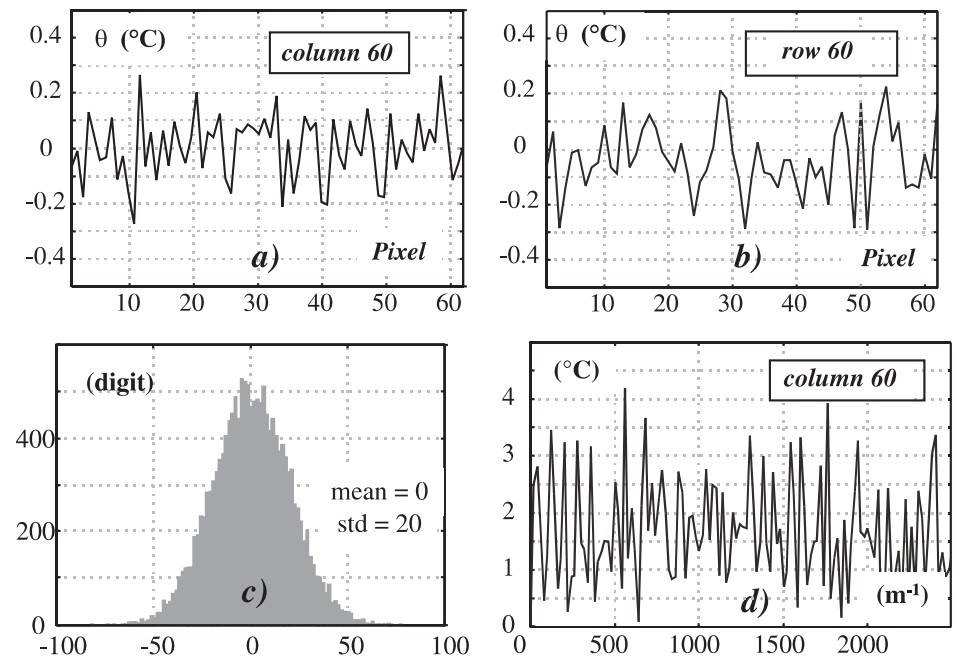

Fig. 3. (a) and (b) longitudinal and transversal noise profiles; (c) histogram showing, for an image, the probability density of noise (digitised level); (d) amplitude spectrum for a column of noise: white noise. 
filtering and the space derivatives estimates are performed using a Gaussian filter. The kernel corresponding to the discrete filter can expressed as

$$
\phi(r)=\frac{v_{\mathrm{c}}}{\sqrt{\pi}} \exp \left(-v_{\mathrm{c}}^{2} \cdot r^{2}\right),
$$

where $v_{\mathrm{c}}$ is related to the cut-off frequency and where $r=x_{3}$ or $r=\|\mathbf{x}\|$ for the 1D and 2D problems, respectively. To estimate the Laplacian of the filtered temperature, the second derivatives of the continuous kernel were classically used [20]. They read:

$$
\begin{aligned}
& \Delta \phi(r)=\frac{4 v_{\mathrm{c}}^{5}}{\sqrt{\pi}} \exp \left(-v_{\mathrm{c}}^{2} \cdot r^{2}\right) \cdot\left(r^{2}-\frac{1}{2 v_{\mathrm{c}}^{2}}\right) \quad(1 \mathrm{D}), \\
& \Delta \phi(r)=\frac{4 v_{\mathrm{c}}^{6}}{\sqrt{\pi}} \exp \left(-v_{\mathrm{c}}^{2} \cdot r^{2}\right) \cdot\left(r^{2}-\frac{1}{v_{\mathrm{c}}^{2}}\right) \quad(2 \mathrm{D}) .
\end{aligned}
$$

These operations were traditionally realised using FFT or DFT to limit the computational time [21]. The filtered temperature data $\bar{\theta}_{\mathrm{f}}$ and the filtered temperature Laplacian $\Delta \bar{\theta}_{\mathrm{f}}$ can be formally written as

$$
\begin{aligned}
& \bar{\theta}_{\mathrm{f}}=\operatorname{Re}\left[\mathscr{F}_{d}^{-1}\left(N \mathscr{F}_{d}(\bar{\theta}) \cdot \mathscr{F}_{d}(\phi)\right)\right], \\
& \Delta \bar{\theta}_{\mathrm{f}}=\operatorname{Re}\left[\mathscr{\mathscr { F }}_{d}^{-1}\left(N \mathscr{\mathscr { F }}_{d}(\bar{\theta}) \cdot \mathscr{\mathscr { F }}_{d}(\Delta \phi)\right)\right],
\end{aligned}
$$

where $N$ is the number of data of the extended profile or extended image while Re stands for the real part of the complex data.

The time derivative of the temperature can also be estimated by using a time convolutive filtering or, more directly, by using a finite differences approximation. If we note $\dot{\bar{\theta}}_{\mathrm{f}}$ this estimate, on the basis of Eqs. (4.8a), (4.15a)-(5.16), the heat sources is locally determined by the relation

$$
\rho C_{\varepsilon, \alpha}\left(\dot{\bar{\theta}}_{\mathrm{f}}+\frac{\bar{\theta}_{\mathrm{f}}}{\tau_{\text {th }}}\right)-k \Delta \bar{\theta}_{\mathrm{f}}=\bar{s},
$$

where $\tau_{\text {th }}$ symbolises a time constant related to the heat losses. In the 1D problem, using Eq. (4.8a), this constant reads

$$
\tau_{\mathrm{th}}^{1 \mathrm{D}}=\frac{\rho C_{\varepsilon, \alpha} S}{2 h(e+l)}
$$

and characterises the lateral heat exchanges between the sample and the surrounding air. For 2D problem, using Eq. (4.15a), the constant becomes 


$$
\tau_{\mathrm{th}}^{2 \mathrm{D}}=\frac{\rho C_{\varepsilon, \alpha} e}{2 h} .
$$

This constant is related to the heat losses perpendicular to the specimen surface.

\section{Checking the image processing}

To check the efficiency of the data processing, numerical simulations are performed in cases where analytical solutions exist. For all examples shown below, a heat source distribution is first chosen. Then, using either (4.8a)-(4.8c) or (4.15a)-(4.15d) partial derivative problem, the analytical solutions are derived from a spectral resolution. The solution $\bar{\theta}\left(x_{3}, t\right)$ (or $\left.\bar{\theta}(\mathbf{x}, t)\right)$ is expressed in the spectral base of the spatial partial derivative operator, composed by the eigen functions that are compatible with the boundary conditions (4.8b) or (4.15b) and (4.15c). The reader, interested in the mathematical background of this approach, can consult [22]. A noise is then added to the temperature, either by using a random function to simulate noisy experimental data, or more directly, by using experimental noised images. A heat source distribution can finally be deduced from such a data set by the mean of Eq. (5.17), and compared to the given initial distribution. The space-time discretisation steps, the thermophysical parameters and the heat exchange coefficients are chosen, of course, close to the ones of the experimental tests.

\subsection{Examples of $1 D$ thermal diffusion problem}

\subsubsection{A non-homogeneous case}

Under certain regularity and 'projectability' conditions, the heat source $\bar{s}\left(x_{3}, t\right)$ and the temperature variations $\bar{\theta}\left(x_{3}, t\right)$ can be written in the case of Eqs. (4.8a)-(4.8c) as

$$
\begin{aligned}
& \bar{s}\left(x_{3}, t\right)=\sum_{p=0}^{\infty}\left[s_{p}(t) \cos \left(\omega_{p} x_{3}\right)+S_{p}(t) \sin \left(\Omega_{p} x_{3}\right)\right], \\
& \bar{\theta}\left(x_{3}, t\right)=\sum_{p=0}^{\infty}\left[\theta_{p}(t) \cos \left(\omega_{p} x_{3}\right)+\Theta_{p}(t) \sin \left(\Omega_{p} x_{3}\right)\right]
\end{aligned}
$$

where the functions $\cos \left(\omega_{p} x_{3}\right)$ and $\sin \left(\Omega_{p} x_{3}\right)$ for $p=0,1, \ldots, \infty$ represent the eigen functions base. The eigen pulsations $\omega_{p}$ and $\Omega_{p}$ are respectively deduced from the roots $r_{p}$ and $R_{p}$ of the following equations derived from the boundary conditions:

$$
\tan \left(\pi r_{p}\right)-\frac{\lambda_{1} L}{2 \pi r_{p}}=0, \quad \tan \left(\pi R_{p}\right)-\frac{2 \pi R_{p}}{\lambda_{1} L}=0,
$$

by relations

$$
\omega_{p}=\frac{2 \pi r_{p}}{L}, \quad \Omega_{p}=\frac{2 \pi R_{p}}{L} .
$$


The classical literature gives many examples of thermal problems solved by spectral methods [23]. In our situation, we can nevertheless notice some slight originality: in the framework of finite transformations of solids, the length $L$ of the sample must be considered as a function of time. The eigen pulsations also become time dependent. During strain-controlled tensile test, the length $L(t)$ is given, the values $\omega_{p}\left(t_{k}\right)$ and $\Omega_{p}\left(t_{k}\right)$ at any instant $t_{k}$ can then be deduced. The functions $s_{p}(t)$, $S_{p}(t), \theta_{p}(t)$ and $\Theta_{p}(t)$ are the projections of $\bar{s}\left(x_{3}, t\right)$ and $\bar{\theta}\left(x_{3}, t\right)$ on the eigen functions respectively

$$
\begin{aligned}
s_{p}(t)=\frac{\left\langle\bar{s}\left(x_{3}, t\right) \mid \cos \left(\omega_{p}(t) x_{3}\right)\right\rangle}{\left\langle\cos \left(\omega_{p}(t) x_{3}\right) \mid \cos \left(\omega_{p}(t) x_{3}\right)\right\rangle}, & S_{p}(t)=\frac{\left\langle\bar{s}\left(x_{3}, t\right) \mid \sin \left(\Omega_{p}(t) x_{3}\right)\right\rangle}{\left\langle\sin \left(\Omega_{p}(t) x_{3}\right) \mid \sin \left(\Omega_{p}(t) x_{3}\right)\right\rangle}, \\
\theta_{p}(t)=\frac{\left\langle\bar{\theta}\left(x_{3}, t\right) \mid \cos \left(\omega_{p}(t) x_{3}\right)\right\rangle}{\left\langle\cos \left(\omega_{p}(t) x_{3}\right) \mid \cos \left(\omega_{p}(t) x_{3}\right)\right\rangle}, & \Theta_{p}(t)=\frac{\left\langle\bar{\theta}\left(x_{3}, t\right) \mid \sin \left(\Omega_{p}(t) x_{3}\right)\right\rangle}{\left\langle\sin \left(\Omega_{p}(t) x_{3}\right) \mid \sin \left(\Omega_{p}(t) x_{3}\right)\right\rangle},
\end{aligned}
$$

where the scalar product $\langle u(x) \mid v(x)\rangle$ is defined by $\int_{-L / 2}^{L / 2} u(\xi) v(\xi) \mathrm{d} \xi$. If we note:

$$
\begin{aligned}
& A_{p, q}(t)=\frac{\left\langle x_{3} \sin \left(\omega_{p}(t) x_{3}\right) \mid \cos \left(\omega_{p}(t) x_{3}\right)\right\rangle}{\left\langle\cos \left(\omega_{p}(t) x_{3}\right) \mid \cos \left(\omega_{p}(t) x_{3}\right)\right\rangle} \omega_{p}, \\
& B_{p, q}(t)=\frac{\left\langle x_{3} \cos \left(\Omega_{p}(t) x_{3}\right) \mid \sin \left(\omega_{p}(t) x_{3}\right)\right\rangle}{\left\langle\sin \left(\omega_{p}(t) x_{3}\right) \mid \sin \left(\omega_{p}(t) x_{3}\right)\right\rangle} \Omega_{p},
\end{aligned}
$$

the components of matrices $\mathbf{A}$ and $\mathbf{B}$, respectively, if $d_{\mathrm{th}}=k / \rho C_{\varepsilon, \alpha}$ and if

$$
d_{p}(t)=\frac{1}{\tau_{\mathrm{th}}}+d_{\mathrm{th}} \omega_{p}(t), \quad D_{p}(t)=\frac{1}{\tau_{\mathrm{th}}}+d_{\mathrm{th}} \Omega_{p}(t)
$$

denote the diagonal components of matrices $\mathbf{d}(t)$ and $\mathbf{D}(t)$, respectively, then, the vectors $\boldsymbol{\theta}(t)$ of components $\theta_{p}(t)$ and $\boldsymbol{\Theta}(t)$ of components $\Theta_{p}(t)$ are solutions of the following first order differential systems:

$$
\begin{aligned}
& \dot{\boldsymbol{\theta}}(t)+[\mathbf{A}(t)+\mathbf{d}(t)] \boldsymbol{\theta}(t)=\mathbf{s}(t), \\
& \boldsymbol{\theta}(0)=\mathbf{0},
\end{aligned}
$$

and

$$
\begin{aligned}
& \dot{\boldsymbol{\Theta}}(t)+[\mathbf{B}(t)+\mathbf{D}(t)] \boldsymbol{\Theta}(t)=\mathbf{S}(t), \\
& \boldsymbol{\Theta}(0)=\mathbf{0},
\end{aligned}
$$

where $\mathbf{s}(t)$ is the vector of components $s_{p}(t)$ while $\mathbf{S}(t)$ is the vector of components $S_{p}(t)$. 
The choice of the heat source distribution $\bar{s}\left(x_{3}, t\right)$ was motivated by the three following goals: - determining semi-analytic solutions of problem (4.8a)-(4.8c) to compute, with a great accuracy, a temperature field related to a given distribution of heat sources;

- checking the efficiency of the data processing when a localisation of the dissipated energy occurs;

- testing the influence of the thermal noise on the heat source detection.

Numerous examples were analysed. Hereafter, we present a penalising case that shows the robustness of the image processing. To get a first and simple insight of the numerical results, profiles of heat sources have been gathered in a 1D space-time chart. To further simplify the figure interpretation, contour plots have been chosen to quickly visualise the data. The level curves are numbered, note that the heat sources have been divided by the volume heat capacity so that the unit becomes $\left({ }^{\circ} \mathrm{C} \mathrm{s}^{-1}\right)$. The different coefficients used in this example are gathered in Table 1 .

During the first part of the test, (see Figs. 4 and 9, curve a), the chosen heat sources distribution has a triangle shape that moves along a segment representing the length of the sample. This triangle keeps a constant form and its displacement velocity has a sinusoidal evolution. This academic example has been chosen to simulate the case of a heat sources localisation. Conversely, during the second part of the simulation, the heat sources distribution remains spatially uniform to test more particularly the boundary effects. The level curves mark the displacement of the localised triangular source and then indicates a homogeneous evolution of the heat sources. The corresponding temperature chart is plotted in Fig. 5.

A first check of the data processing was performed on the calculated temperatures derived from the spectral solution. The result is shown in Fig. 6 and it is now possible to compare this new heat

Table 1

Geometric and thermophysical constants used in the 1D simulations

\begin{tabular}{llll}
\hline Geometry & $L_{0}=50.10^{-3} \mathrm{~m}$ & $l_{0}=10.10^{-3} \mathrm{~m}$ & $e_{0}=21.10^{-4} \mathrm{~m}$ \\
Material & $\rho=7860 \mathrm{~kg} \mathrm{~m}^{-3}$ & $C_{\varepsilon, \alpha}=472 \mathrm{~J} \mathrm{~kg}^{-1}{ }^{\circ} \mathrm{C}^{-1}$ & $k=63 \mathrm{~W} \mathrm{~m}^{-1}{ }^{\circ} \mathrm{C}^{-1}$ \\
Thermal & $\lambda_{2}=20 \mathrm{~m}^{-1}$ & $\tau_{\mathrm{th}}^{1 \mathrm{D}}=19 \mathrm{~s}$ & 100 Eigen vectors \\
\hline
\end{tabular}

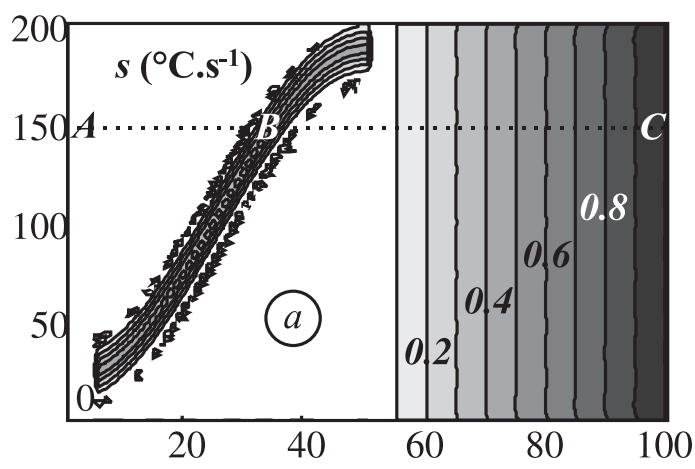

Fig. 4. Space-time distribution of heat sources for a $1 \mathrm{D}$ data processing. The $X$-axis is related to time (image number) and the $Y$-axis to the sample length (pixel). The heat sources are divided by the volume heat capacity. The time evolution of the source along the profile A-B-C corresponds to the curve a of Fig. 9. 


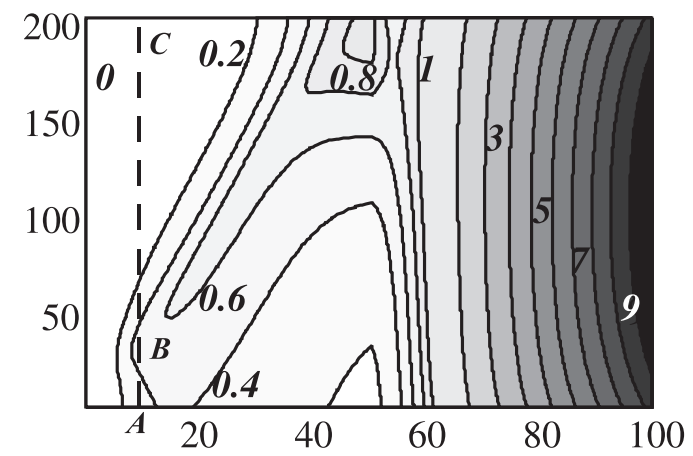

Fig. 5. Temperature chart corresponding to Fig. 4. Because of the heat diffusion, the decomposition of the test in two parts is no longer obvious. The space evolution of the temperature along the profile A-B-C is plotted in Fig. 7.

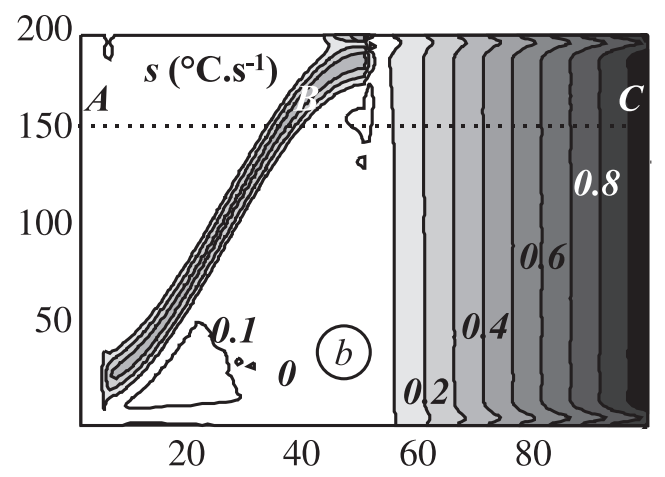

Fig. 6. Space-time distribution of heat sources deduced from the temperature data plotted in Fig. 5. The time evolution of the source along the profile A-B-C corresponds to the curve b of Fig. 9.

sources distribution with the given initial one. The quality of this inverse passage can also be checked in Fig. 9 by comparing curves a and b. The curve b shows the effect of the low-pass convolutive filtering on the heat source determination. Even if no thermal noise disturbs the data, a finite sampling rate leads to a finite cut-off frequency for the discrete filter. This implies an attenuation of the heat sources intensity and a spreading out of the zones where they concentrate.

It is then possible to superimpose a Gaussian noise on the calculated temperature data. In this example, the chosen noise amplitude reaches up to $0.3^{\circ} \mathrm{C}$ of temperature variation. The noisy thermoprofile corresponding to the axis $A-B-C$ defined in Fig. 5 is plotted in Fig. 7.

Using these noisy data, a second check of the image processing was performed. The chart of heat sources is drawn in Fig. 8. To avoid any numerical instability during the image processing, the cut-off frequency of the filter was reduced. As previously, we observed an attenuation of the intensity of the localised heat sources compared to the initial data as well as a spreading out of the zones where they concentrate. However, in spite of the noise we estimate that the results are satisfactory (see Fig. 9). 


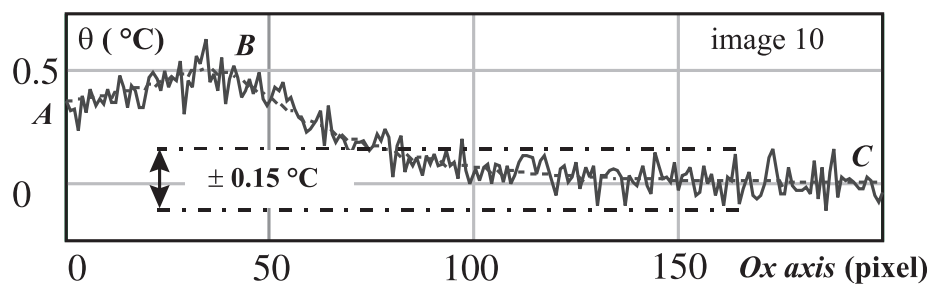

Fig. 7. Calculated and noisy thermoprofiles corresponding to the axis defined in Fig. 5. The noise is chosen here three times as big as the experimental one. For 1D thermal diffusion problem, the noise on data is naturally reduced because the thermoprofile is averaged along the sample width.

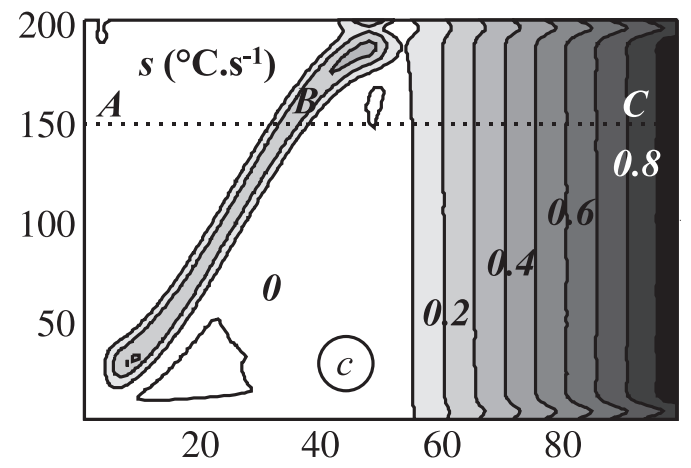

Fig. 8. Space-time distribution of heat sources deduced from the noisy temperature data. The time evolution of the source along the profile A-B-C corresponds to the curve $\mathrm{c}$ of Fig. 9.

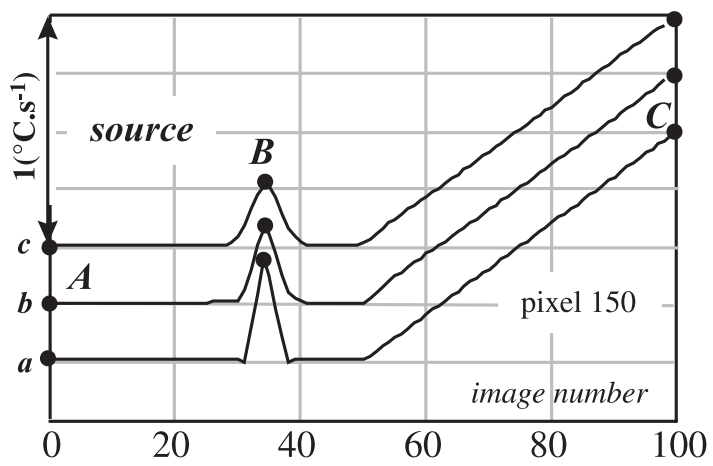

Fig. 9. Heat sources profiles corresponding to the time evolution at the pixel 150. The curve a is associated with the distribution initially given. The curve $b$ represents data directly deduced from the analytic solution of the temperature. The curve c corresponds to data deduced from the noisy temperatures. Naturally, the curves were shifted to facilitate their comparison.

\subsubsection{A particularly important case: the uniform distribution of sources}

If the heat sources are assumed to be uniform, the data processing can be considerably simplified. For instance, let us take the following example where the homogeneous heat source $\bar{s}_{\text {theo }}(t)$ 


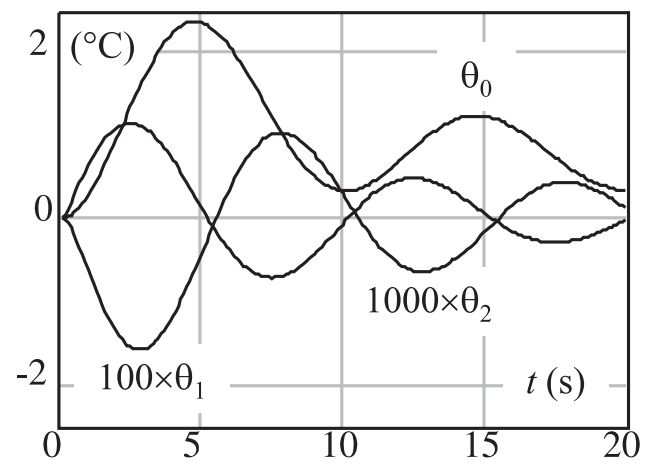

Fig. 10. If the heat source distribution is spatially uniform and when boundary conditions are symmetric and linear, the amplitude of eigen functions decreases rapidly with $p$ as shown above for $p=0,1,2$.

is a deadened sine: $\bar{s}_{\text {theo }}(t)=\sin (\pi / 5 t) \times \exp (-t / 10)$. Keeping the same symmetric and linear boundary conditions and the same geometrical parameters as in the previous example (Table 1), we observe that almost all the temperature signal is concentrated on the first eigen function $\cos \left(\omega_{0} x_{3}\right)$. Fig. 10 shows the strong decrease of the $\theta_{p}$ s amplitudes, the $\Theta \mathrm{s}$ vanishing for even temperature solutions. Such an approximation yields thermal conduction losses directly proportional to the temperature variations.

As a consequence, the convolutive filtering step of the Laplacian operator is no longer to do. The heat source distribution can be directly estimated by

$$
\frac{\tilde{s}\left(x_{3}, t\right)}{\rho C_{\varepsilon, \alpha}}=\left(\frac{\omega_{0} L+\sin \left(\omega_{0} L\right)}{4 \sin \left(\omega_{0} L / 2\right) \cos \left(\omega_{0} x_{3}\right)}\right) \cdot\left(\dot{\tilde{\theta}}\left(x_{3}, t\right)+\frac{\tilde{\theta}\left(x_{3}, t\right)}{\tau_{\mathrm{eq}}}\right) .
$$

The first term of the right-hand member of Eq. (6.11) is a factor close to 1 while $\omega_{0} L \ll 1$. This factor rectifies the approximation done by considering the first eigen function. The equivalent time constant $\tau_{\text {eq }}$ is defined by

$$
\tau_{\mathrm{eq}}=\left(\frac{1}{\tau_{t h}}+d_{\mathrm{th}} \omega_{0}^{2}\right)^{-1} .
$$

In Fig. 11, the evolution of the sources, evaluated in the middle and at one extremity of the sample, can be compared with the theoretical sources. Using the filtered temperature profile and the Eq. (6.11), we find a homogeneous distribution of sources again. In order to be convinced, the absolute error between theoretical and calculated solutions is also plotted.

\subsection{Example of $2 D$ thermal diffusion problem}

In this section, the method is extended to $2 \mathrm{D}$ diffusion problems. As previously, under certain regularity and 'projectability' conditions, in the case of problem (4.15), the heat source $\bar{s}(\mathbf{x}, t)$ and the temperature variations $\bar{\theta}(\mathbf{x}, t)$ can be written as: 


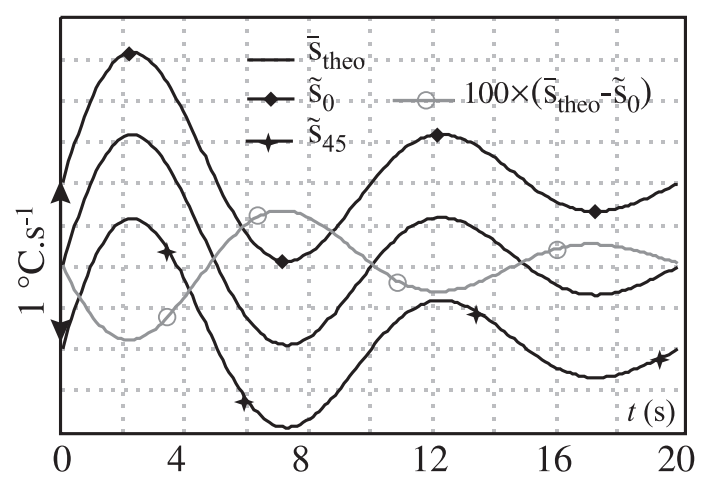

Fig. 11. The evolution of heat sources is plotted for two particular points: one is placed in the middle of the profile while the second is at one extremity. These evolutions can be compared to the theoretical solution. The difference between theoretical and calculated data is also plotted. Of course, the different curves were shifted for a better comparison.

$$
\begin{aligned}
\bar{s}(\mathbf{x}, t)=\sum_{p, q} & {\left[s_{1_{p q}}(t) \cos \left(\omega_{p} x_{2}\right) \cos \left(w_{q} x_{3}\right)+s_{2_{p q}}(t) \cos \left(\omega_{p} x_{2}\right) \sin \left(W_{q} x_{3}\right)\right] } \\
& +\sum_{p, q}\left[s_{3_{p q}}(t) \sin \left(\Omega_{p} x_{2}\right) \cos \left(w_{q} x_{3}\right)+s_{4_{p q}}(t) \sin \left(\Omega_{p} x_{2}\right) \sin \left(W_{q} x_{3}\right)\right], \\
\bar{\theta}(\mathbf{x}, t)= & \sum_{p, q}\left[\theta_{1_{p q}}(t) \cos \left(\omega_{p} x_{2}\right) \cos \left(w_{q} x_{3}\right)+\theta_{2_{p q}}(t) \cos \left(\omega_{p} x_{2}\right) \sin \left(W_{q} x_{3}\right)\right] \\
& +\sum_{p, q}\left[\theta_{3_{p q}}(t) \sin \left(\Omega_{p} x_{2}\right) \cos \left(w_{q} x_{3}\right)+\theta_{4_{p q}}(t) \sin \left(\Omega_{p} x_{2}\right) \sin \left(W_{q} x_{3}\right)\right]
\end{aligned}
$$

where the functions

$$
\begin{aligned}
& V_{1_{p q}}(\mathbf{x})=\cos \left(\omega_{p} x_{2}\right) \cos \left(w_{q} x_{3}\right), \quad V_{2_{p q}}(\mathbf{x})=\cos \left(\omega_{p} x_{2}\right) \sin \left(W_{q} x_{3}\right), \\
& V_{3_{p q}}(\mathbf{x})=\sin \left(\Omega_{p} x_{2}\right) \cos \left(w_{q} x_{3}\right), \quad V_{4_{p q}}(\mathbf{x})=\sin \left(\Omega_{p} x_{2}\right) \sin \left(W_{q} x_{3}\right),
\end{aligned}
$$

for $p, q=0,1, \ldots,+\infty$ constitute the eigen functions base. The eigen pulsations $\omega_{p}, \Omega_{p}, w_{q}$ and $W_{q}$ are deduced from the roots $\rho_{p}, P_{p}, r_{q}$ and $R_{q}$ of the following equations, respectively:

$$
\begin{aligned}
& \tan \left(\pi \rho_{p}\right)-\frac{\lambda_{1} l}{2 \pi \rho_{p}}=0, \quad \tan \left(\pi P_{p}\right)-\frac{2 \pi P_{p}}{\lambda_{1} l}=0, \\
& \tan \left(\pi r_{q}\right)-\frac{\lambda_{2} L}{2 \pi r_{q}}=0, \quad \tan \left(\pi R_{q}\right)-\frac{2 \pi R_{q}}{\lambda_{2} L}=0
\end{aligned}
$$

by the relations $\omega_{p}=2 \pi \rho_{p} / 1, \Omega_{p}=2 \pi P_{p} / l, w_{q}=2 \pi r_{q} / L$ and $W_{q}=2 \pi R_{q} / L$. 
For the sake of simplicity we shall assume, in what follows, the small perturbation hypothesis. In such a situation, Eulerian configuration is mixed up with that of Lagrange and the eigen pulsation can be considered as time constants.

The functions $s_{i_{p q}}(t)$ and $\theta_{i_{p q}}(t)$ for $i=1, \ldots, 4$ are respectively the projections of $\bar{s}(\mathbf{x}, t)$ and $\bar{\theta}(\mathbf{x}, t)$ on the eigen functions $V_{i_{p q}}(\mathbf{x})$ :

$$
s_{i_{p q}}(t)=\frac{\left\langle\bar{s}(\mathbf{x}, t) \mid V_{i_{p q}}(\mathbf{x})\right\rangle}{\left\langle V_{i_{p q}}(\mathbf{x}) \mid V_{i_{p q}}(\mathbf{x})\right\rangle}, \quad \theta_{i_{p q}}(t)=\frac{\left\langle\bar{\theta}(\mathbf{x}, t) \mid V_{i_{p q}}(\mathbf{x})\right\rangle}{\left\langle V_{i_{p q}}(\mathbf{x}) \mid V_{i_{p q}}(\mathbf{x})\right\rangle},
$$

where the scalar product $\langle u(\mathbf{x}) \mid v(\mathbf{x})\rangle$ is defined by

$$
\langle u(\mathbf{x}) \mid v(\mathbf{x})\rangle=\int_{-l / 2}^{l / 2} \int_{-L / 2}^{L / 2} u(\mathbf{x}) \cdot v(\mathbf{x}) \mathrm{d} \xi \mathrm{d} \chi .
$$

Now, if we note:

$$
\begin{aligned}
& \Omega_{1_{p q}}^{2}=\omega_{p}^{2}+w_{q}^{2}, \\
& \Omega_{2_{p q}}^{2}=\omega_{p}^{2}+W_{q}^{2}, \\
& \Omega_{3_{p q}}^{2}=\Omega_{p}^{2}+w_{q}^{2}, \\
& \Omega_{4_{p q}}^{2}=\Omega_{p}^{2}+W_{q}^{2},
\end{aligned}
$$

then, each function $\theta_{i_{p q}}(t)$ verifies the following differential equation

$$
\dot{\theta}_{i_{p q}}(t)+\left(d_{\mathrm{th}} \Omega_{i_{p q}}^{2}+\frac{1}{\tau_{\mathrm{th}}}\right) \cdot \theta_{i_{p q}}(t)=s_{i_{p q}}(t)
$$

with $\theta_{i_{p q}}(0)=0$.

The distribution of the heat sources, chosen to illustrate the method, takes a pyramidal form (2D extension of the preceding case). In the course of time, the pyramid keeps a constant form and moves on a plan. With this distribution, spatially heterogeneous (the pyramid), one superimposes a spatially homogeneous but non stationary heat source (the plan). The different coefficients used in this example are gathered in Table 2.

An illustration of the results obtained by image processing is proposed in Fig. 12. One considers the moment corresponding to the image 12. From the given distribution of sources (Fig. 12), one deduces by spectral method the temperatures charts (Fig. 13). For this example, $20 \times 20$ eigen vectors were used. Then, the estimate of the first member of the Eq. (5.17) by DFT makes it possible to rebuild a field of sources. The results obtained for three levels of noise (Fig. 14) are shown in Figs. 15-18. 
Table 2

Geometric and thermophysical constants used in the 2D simulations

\begin{tabular}{llll}
\hline Geometry & $L_{0}=33.10^{-3} \mathrm{~m}$ & $l_{0}=22.10^{-3} \mathrm{~m}$ & $e_{0}=2.10^{-3} \mathrm{~m}$ \\
Material & $\rho=7860 \mathrm{~kg} \mathrm{~m}^{-3}$ & $C_{\varepsilon, \alpha}=480 \mathrm{~J} \mathrm{~kg}^{-1}{ }^{-1} \mathrm{C}^{-1}$ & $k=60 \mathrm{~W} \mathrm{~m}{ }^{-1} \mathrm{C}^{-1}$ \\
Thermal & $\lambda_{1}=0.2 \mathrm{~m}^{-1}$ & $\lambda_{2}=20 \mathrm{~m}^{-1}$ & $\tau_{\text {th }}^{2 \mathrm{D}}=314 \mathrm{~s}$ \\
\hline
\end{tabular}

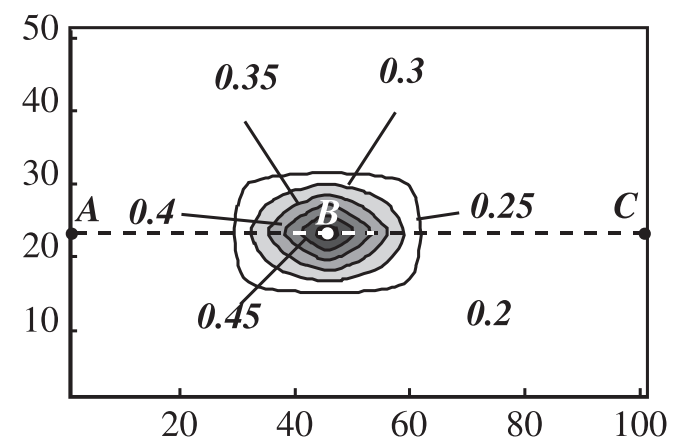

Fig. 12. Graph of analytic distribution of heat sources corresponding to image 12. Divided by the volume heat capacity, the unit of the heat sources becomes $\left({ }^{\circ} \mathrm{C} \mathrm{s}^{-1}\right)$.

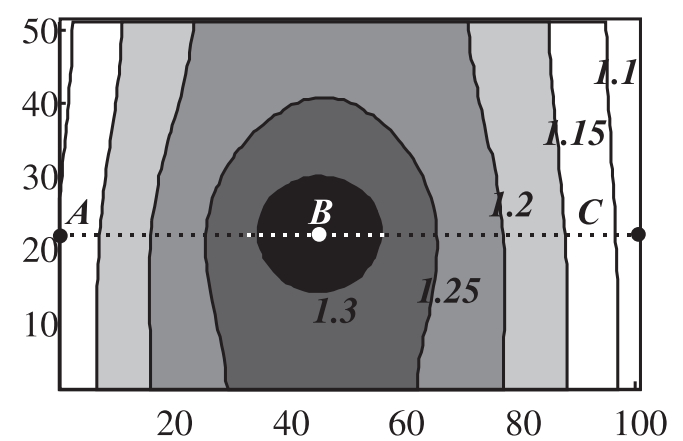

Fig. 13. Graph of the analytic temperature solution corresponding to image 12 . The unit is ${ }^{\circ} \mathrm{C}$. A longitudinal profile $\mathrm{A}-\mathrm{B}-\mathrm{C}$ is also plotted. It has been chosen in Fig. 14 to visualise the different noise levels, the thermal gradients and the curvatures intensities.

Some light differences can be noticed between analytic solution of heat sources and those deduced from temperature maps determined by spectral method. These differences develop very quickly with the noise amplitude. As previously, the errors come mainly from the presence of the Laplacian in the equation of heat. To limit these errors, it is necessary to decrease the cut-off frequency of the filter, on the other hand, one observes, as it should be, a fall of the sources intensity, and a spreading out of the zones where the sources concentrate (elimination of the high spatial frequencies). 

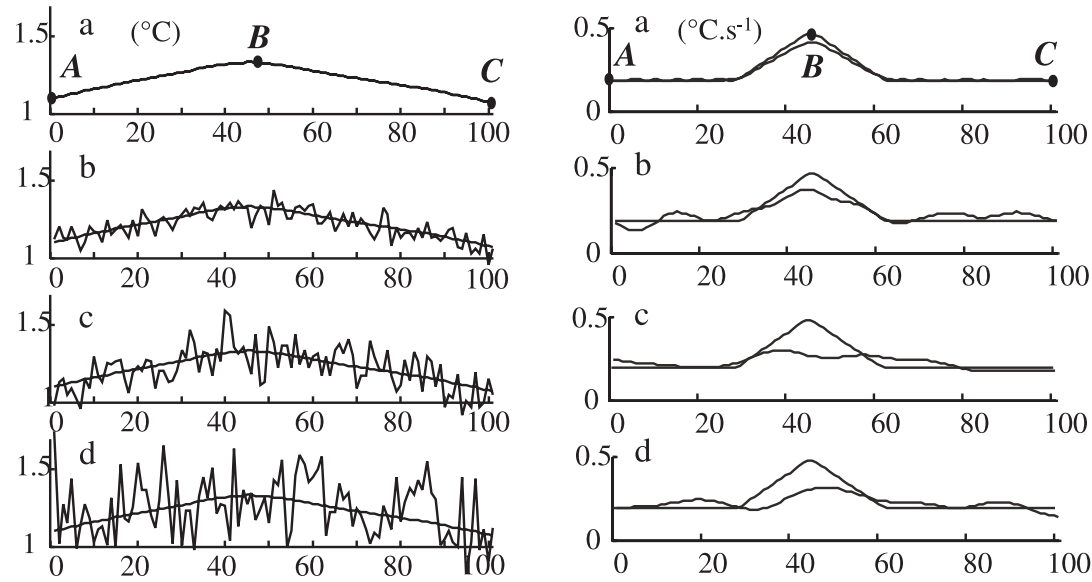

Fig. 14. Increasing noise levels shown on the A-B-C profile defined in Fig. 13 (left). Corresponding heat sources (right): (a) $\mathrm{std}=0^{\circ} \mathrm{C}$; (b) $\mathrm{std}=0.2^{\circ} \mathrm{C}$; (c) $\mathrm{std}=0.4^{\circ} \mathrm{C}$; (d) $\mathrm{std}=0.6^{\circ} \mathrm{C}$. For the three levels of noise the mean value equals zero and std stands for the standard deviation.

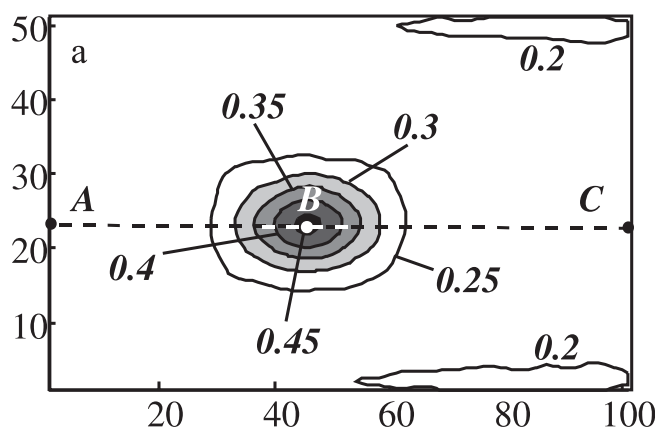

Fig. 15. Graph of the heat sources distribution related to image 12 when no noise disrupts the temperature field.

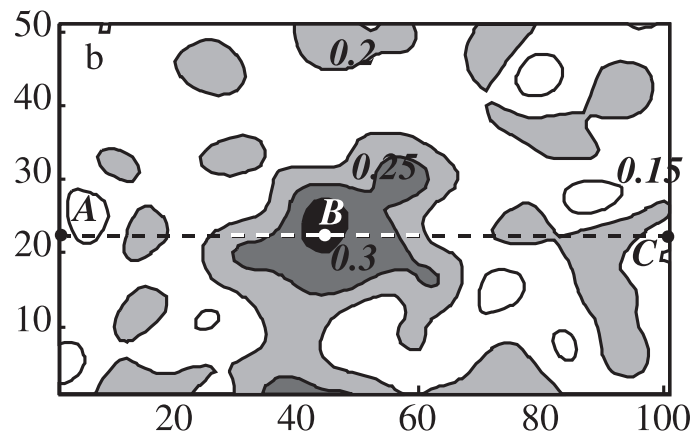

Fig. 16. Graph of the heat sources distribution related to image 12 for noise of type $b$. 


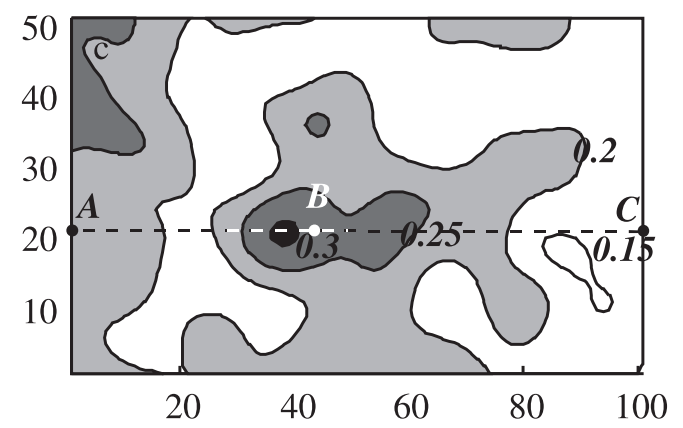

Fig. 17. Graph of the heat sources distribution related to image 12 for noise of type c.

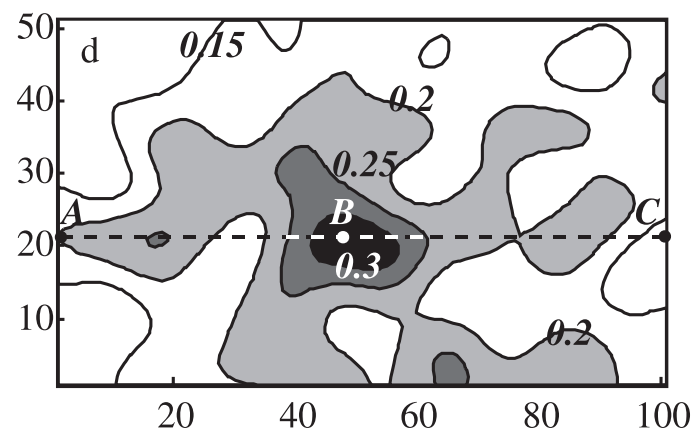

Fig. 18. Graph of the heat sources distribution related to image 12 for noise of type d.

\section{Application to strain localisation}

When a structure is mechanically loaded, its deformation passes more or less suddenly from a diffuse mode characterised by regular strain fields to localised modes defined by zones where the strains develop and concentrate. The research fields related to localisation phenomena are very active. Many authors have worked and still work today on the experimental, theoretical and numerical aspects of such deformation mechanisms. In the bibliography references, we have mentioned just a small part of the very large diversity of scientific approaches proposed in the literature. In the particular case of the plastic or viscoplastic materials, the studies have tried to take account of the large variety of loading and have naturally focused on the kinematic aspects of the localisation. Let us mention for instance the works of Considère [24], Hill [25], Mandel [26], Hart [27], Rice [28], and more recently, in France, Benallal [29] and Fressengeas and Molinari [30]. When the material behaviour is time-independent, the localisation phenomenon is to appear as soon as a bifurcated solution to the linearised problem exist. This bifurcation can then be physically translated by a spatial discontinuity of the strain rate field. If the behaviour is timedependent, a perturbation analysis of the linearised problem is often proposed. In such a case, the localisation is related to an instability, the perturbation amplitude does not diminish but on the 
contrary increases. Other works as those done by Estrin [31], Neuhauser [32], Zaizer [33], describe the microstructural origins of localisation phenomena that sometimes occur under the form of Lüders or PLC bands. Sometimes, authors, like Ferron [34] and Marchand, [35], give an account of the thermal effects accompanying the localisation processes. In what follows, the aim is to show that the experimental improvements made in the field of quantitative infrared thermography allow observing the dissipative manifestations related to localisation.

\subsection{Heat evolved during plastic hardening}

In the case of elastoplastic material, the following set of state variables is generally chosen: the absolute temperature $\alpha_{0}=T$, a large strain tensor $\alpha_{1}=\varepsilon$, and $n-1$ internal variables garthered in the vector $\alpha$ characterising the hardening state of the material.

The relative smallness of the thermal dilatability of steels allows to suggest that the heat source due to the thermoelastic couplings becomes rapidly negligible when compared with the intrinsic dissipation $d_{1}$ developed during the elastoplastic transformation. That gives

$$
\rho T \boldsymbol{\psi}_{, \boldsymbol{T}, \varepsilon}: \dot{\boldsymbol{\varepsilon}} \ll d_{1}=\boldsymbol{\sigma}: \mathbf{D}-\rho \boldsymbol{\psi}_{, \varepsilon} \cdot \dot{\boldsymbol{\varepsilon}}-\rho \boldsymbol{\psi}_{, \alpha} \cdot \dot{\alpha} .
$$

In addition, we can reasonably admit that the small temperature variations induced by the deformation process have no influence on the hardening state. As a consequence, the heat sources related to the coupling terms between temperature and the hardening variables can also be neglected

$$
\rho T \psi_{, T, \alpha} \cdot \dot{\alpha} \approx 0 .
$$

Let us recall that this assertion becomes false as soon as a first order phase transformation occurs insofar as $\rho T \psi_{, T, \alpha} \cdot \dot{\alpha}$ represents the latent heat rate [36]. For instance, if a stress-induced martensite transformation occurs, Eq. (7.2) is no longer admissible [37].

\subsection{Experimental results}

Quasi-static and monotone tensile tests were performed on standard thin and flat samples ( 2.5 $\mathrm{mm} \times 12.5 \mathrm{~mm} \times 50 \mathrm{~mm}$ ) [37-39]. The experiments are displacement-controlled and the room temperature is kept constant $(300 \mathrm{~K})$. Several shades of steel, frequently used in metal forming, were chosen. Their European names are S355MC, HR55, DD14. The passage from dissipation to dissipated energy is possible while the displacement fields of the transformation are known as it is the case in the framework of small perturbations (negligible displacement) or homogeneous tension tests (uniform strain fields). In order not to prejudge homogeneity of the sample response, we prefer to present the temporal evolution of the dissipation distribution. Kinematics measurements, carried out using speckle images, supported this careful attitude [40,41]. The normalised evolution of the load were systematically plotted to enable the reader to make a correspondence between the usual and global response of the sample and the local measurements. The maximal amplitude of load is systematically told in the figure legends.

When a plastic plateau exists, a first mode of localisation is observed between the bounds $A$ and $B$. In such cases, one (Fig. 19) or more (Fig. 20) dissipative 'waves' propagate at a constant velocity along the gauge part of the sample. Energetic and kinematic effects have naturally been 

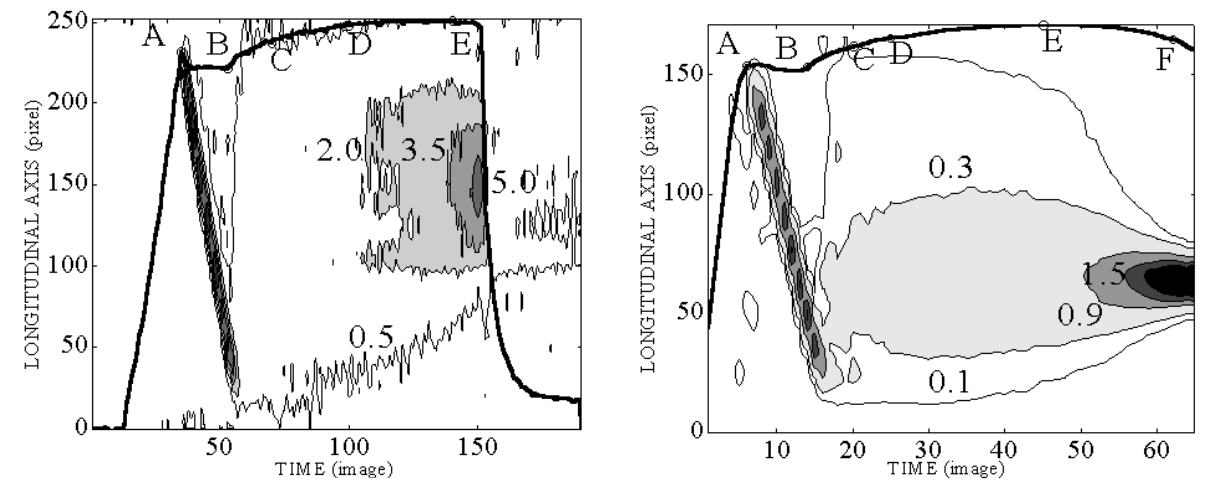

Fig. 19. S355MC steel; lengthwise profiles of the intrinsic dissipation (left) and of the longitudinal component of the Green-Lagrange strain tensor rate (right: after [39]). The dissipation unit is $\mathrm{W} \mathrm{cm}{ }^{-3}$; the strain rate unit is $10^{-2} \mathrm{~s}^{-1}$. The maximum load is $F_{\max }=16.2 \mathrm{kN}$.
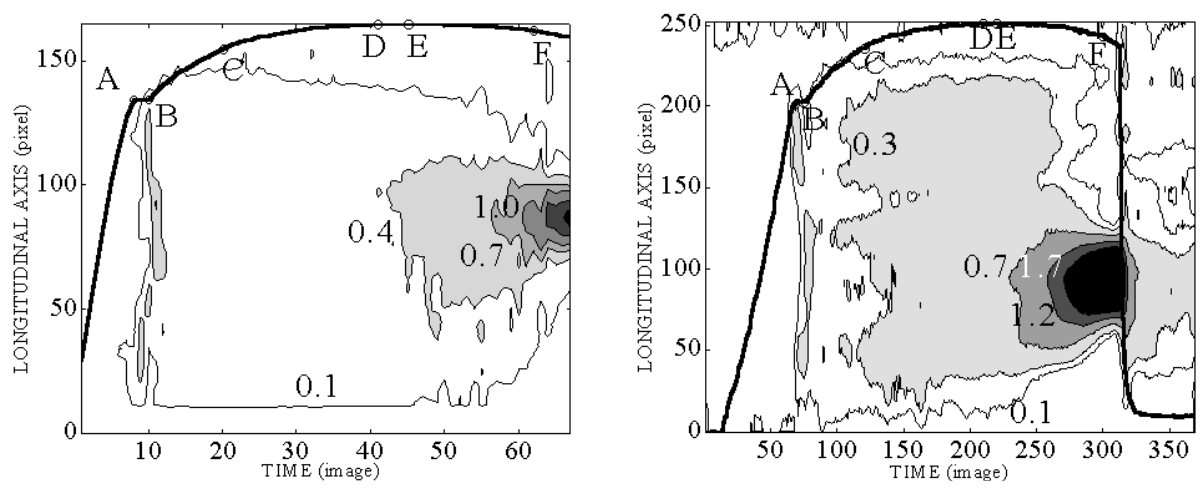

Fig. 20. HR55 steel; lengthwise profiles of the intrinsic dissipation (left) and of the longitudinal component of the Green-Lagrange strain rate tensor (right: after [39]). $F_{\max }=24.5 \mathrm{kN}$.

associated with Lüders band propagation. The angle between the loading axis and the band front during its movement is around $70^{\circ}$. The waves break when strain hardening starts.

At the beginning of the strain hardening (stage B-D), the dissipation field remains approximately uniform, then, stronger dissipation zones with high strain rate appear and concentrate progressively until the maximum of load is reached (stage D-E). During the strain softening (stage E-F), the dissipative and kinematic effects of localisation increase rapidly. In the particular case of S355MC steel, the dissipation chart corresponding to each step previously mentioned is drawn in Fig. 21.

Remember that infrared and speckle tests are for the moment separately performed. As a consequence, energetic and kinematic results are not obtained with the same sample. The loci where necking takes place may naturally be different from one sample to another.

Note that the DD14 load-elongation curve does not present a plastic plateau (Fig. 22); as mentioned above, no band effect has been observed. 
a)

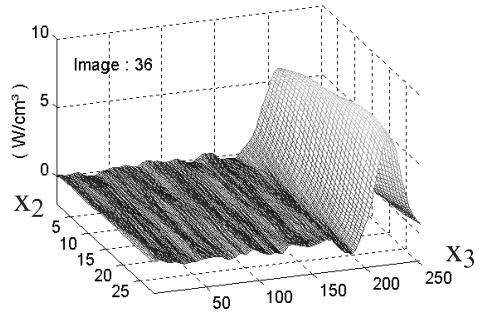

c)

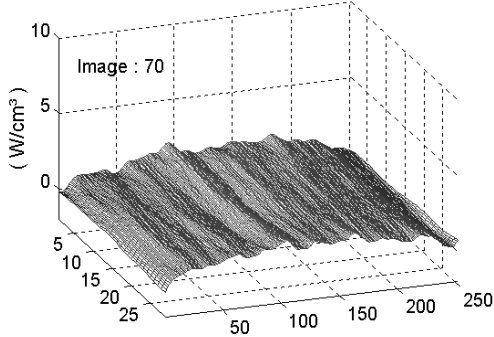

e)

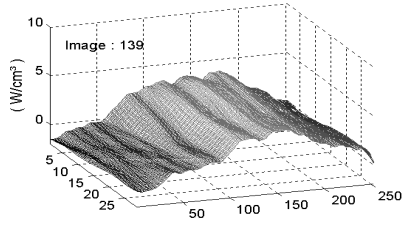

b)

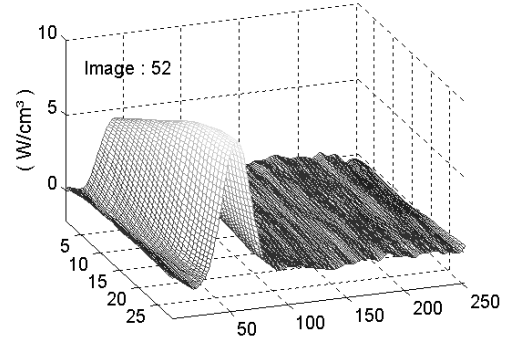

d)
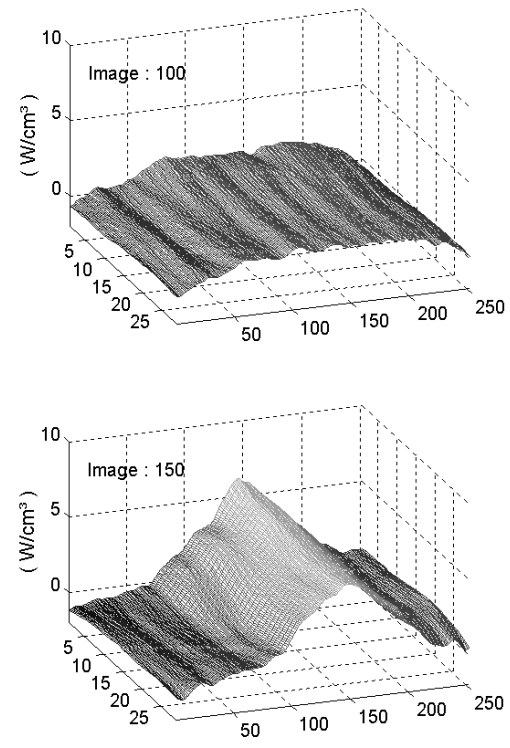

Fig. 21. Dissipation charts in the case of the S355MC steel. The axes are taken respectively in the direction $x_{2}$ and $x_{3}$ of the width and length. Charts (a) and (b) evidence the band propagation. Charts (c)-(f) illustrate the progressive concentration of strong dissipation zones.
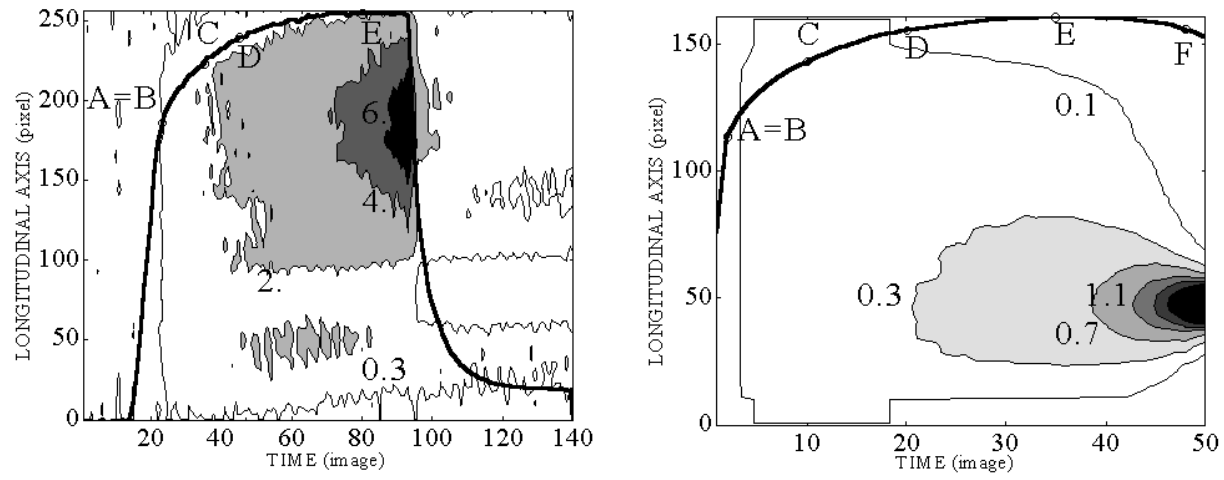

Fig. 22. DD14 steel; lengthwise profile evolution of the intrinsic dissipation (left) and of the longitudinal component of the Green-Lagrange strain rate tensor (right: after [39]). $F_{\max }=13.5 \mathrm{kN}$. 

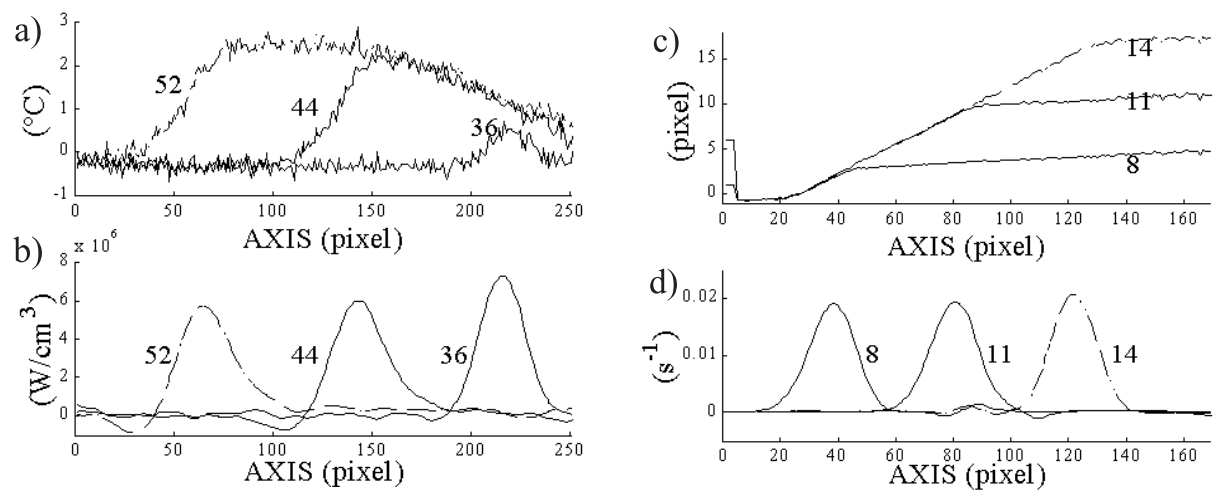

Fig. 23. S355MC Steel; Thermomechanical manifestations related to a Lüders band propagation: (a) temperature, (b) intrinsic dissipation, (c) longitudinal displacement, (d) longitudinal component profiles of the Lagrangian strain rate. Profiles nos. 36, 44 and 52 have been selected from the infrared approach and profiles nos. 8,11 and 14 from the speckle one.

The kinematic and dissipative phenomena related to band propagation are illustrated in detail in Fig. 23 for S355MC steel by plotting several lengthwise profiles between points $A$ and $B$.

\section{Discussion}

This paper aimed to show that thermography techniques could be used with benefit to observe the calorific effects accompanying the localisation phenomena. These techniques propose to pass from temperature information given by the infrared camera to a distribution of heat sources on surface of the steel samples. Sudden and violent manifestations occurring during the plastic plateau have been related to Lüders band propagation, whereas early and progressive features arising during the strain hardening have been interpreted as heraldic signs of the local necking. The main information given by such experimental analyses seems to be the precocity of the heterogeneity development in the macroscopic fields of thermomechanical variables. The gauge part of the sample must be considered as a structure and no longer as an elementary material volume uniformly strained and stressed. This unpalatable fact is currently difficult to reconcile with the theoretical views of localisation since classical formalisms are based on the subtle knowledge of the constitutive equations of the material. These equations are in fact identified on the basis of a range of tests, such as the classical tensile test, for which the existence of homogeneous strain and stress fields is very often implicitly assumed. For the moment, only the pioneering Considère criterion will be briefly evoked here [24] because it concerns the whole specimen. This criterion claims that the necking starts in tension as soon as the maximal load supported by the sample is reached. To make the classical Considère construction the uniaxial 'true' stress is defined as the load divided by the instantaneous sample section which is deduced from the initial section assuming isovolumic and homogeneous transformation. This last assertion is obviously not in agreement with the results shown here. The localisation develops during the hardening of the structure and sometimes just after the yield load. 


\section{References}

[1] Lord Kelvin, On the thermo-elastic and thermo-magnetic properties of matter, Trans. Roy. Soc. Edimb 20 (1853) 161.

[2] L. Kelvin, On the thermo-elastic and thermo-magnetic properties of matter, Math. Phys. Papers 1 (1882) 174.

[3] G.I. Taylor, H. Quinney, The latent energy remaining in a metal after cold working, in: Proceedings of the Royal Society, London, 1934.

[4] R.O. Williams, A deformation calorimeter, Rev. Sci. Inst. 13 (1960) 12.

[5] R.O. Williams, The stored energy of copper at $24^{\circ} \mathrm{C}$, Acta. Met. 13 (1965).

[6] M.B. Bever, D.L. Holt, A.L. Titchener, The stored energy of cold work, in: Christian, Chalmers, Massalski (Eds.), Progress in Materials Science, vol. 17, Pergamon Press, Oxford, 1975.

[7] J. Mandel, Energie élastique et travail dissipé dans les modèles, in: H.D. Bui (Ed.), Dissipation d'énergie dans une déformation plastique, Cahiers Français de Rhéologie, I, 1965.

[8] A. Chrysochoos, J.C. Chezeaux, H. Caumon, Analyse thermomécanique des lois de comportement par thermographie infra-rouge, Rev. de Phys. Appl. 24 (1989) 215-225.

[9] A. Chrysochoos, G. Martin, Tensile test microcalorimetry for thermomechanical behaviour analysis, J. Mat. Sci. Eng. A108 (1989) 25-32.

[10] A. Chrysochoos, F. Belmahjoub, Thermographic analysis of thermo-mechanical couplings, Arch. Mech. 44 (1992) $55-68$.

[11] A. Chrysochoos, H. Pham, O. Maisonneuve, Energy balance of thermoelastic martensite transformation under stress, Nucl. Eng. Design 162 (1996) 1-12.

[12] R. Peyroux, A. Chrysochoos, C. Licht, M. Löbel, Thermomechanical couplings and pseudoelasticity of shape memory alloys, Int. J. Eng. Sci. 36 (4) (1998) 489-509.

[13] H. Callen, Thermodynamics and an Introduction to Thermostatistics, Wiley, New York, 1985.

[14] P. Germain, Mécanique, Ellipses Ed., Ecole Polytechnique, T2, 1986.

[15] J.C. Chezeaux, Application de la radiométrie infrarouge à l'étude des structures mécaniques: numérisation et acquisition de l'image thermique de la caméra AGA SW 780, Rapport DGRST No, 79.7, 1981, p. 1535.

[16] R. Bouc, B. Nayroles, Méthodes et résultats en thermographie infrarouge des solides, J. de Méc. Théo. et Appl. 4 (1985) 27-58.

[17] A Capitana, E. Stavre, Algorithms and convergence results for an inverse problem, Institul de Matematica al Academei Romane, preprint No. 25/1996, ISSN 025 3638, 1996.

[18] A.B. de Vriendt, in: G. Morin (Ed.), Transmission de la chaleur, vol. 1, Tome1 et 2, vol. 2, 1987.

[19] E.O. Brigham, The Fast Fourier Transform, Prentice-Hall, Englewood Cliffs, NJ, 1974, pp. 123-148.

[20] A. Chrysochoos, J.C. Dupre, An infra-red set-up for continuum thermomechanics, in: Quantitative Infrared Thermography, Proceedings of the Eurotherm Seminar No. 27, Ed. Euro. Thermique et Industrie, 1992, pp. 129134.

[21] C. Gasquet, P. Witomski, Analyse de Fourier et Applications, Second ed., Masson, Paris, 1995, pp. 231-305.

[22] C. Soize, Méthodes Mathématiques en Analyse du Signal, Masson, Paris, 1993, pp. 163-206.

[23] H.S. Carslaw, J.C. Jeager, Conduction of Heat in Solids, Clarendon Press, Oxford, 1959.

[24] A. Considère, L'emploi du fer et de l'acier dans les constructions, Annales des Ponts et Chaussées 9 (1885) 574-595.

[25] R. Hill, A general theory of uniqueness and stability in elastic-plastic solids, J. Mech. Phys. Solids 6 (1958) 236-249.

[26] J. Mandel, Condition de stabilité et postulat de Drücker, Rhéologie et Mécanique des sols, in: Kravtchenko et Syries (Ed.), IUTAM Symposium, Grenoble, 1964.

[27] E.W. Hart, Theory of tensile test, Acta Metall. 15 (1967) 351-355.

[28] J.R. Rice, The localisation of plastic deformation, J. Theor. Appl. Mech. 1976.

[29] A. Benallal, On localization phenomena in thermo-elasto-plasticity, Arch. Mech. 44 (1) (1992) 15-29.

[30] C. Fressengeas, A. Molinari, Inertia and thermal effects on the localization of plastic flow, Acta Metall. 33 (1985) 387-396.

[31] Y. Estrin, L.P. Kubin, Plastic instabilities: Classification and physical mechanisms, Res Mechanica 23 (1988) 197-221.

[32] H. Neuhaüser, Collective micro-shear processes and plastic instabilities in crystaline and amorphous structures, Int. J. Plast. 9 (1993) 421-435. 
[33] M. Zaiser, P. Hähner, Oscillatory modes of plastic deformation: theoretical concepts, Phys. Stat. Sol. B 199 (1997) 267-330.

[34] G. Ferron, Influence of heating generation and conduction on plastic stability under uniaxial tension, Mat. Sci. Eng. 49 (1981) 241-248.

[35] A. Marchand, J. Duffy, An experimental study of the formation process of adiabatic shear bands in a structural steel, J. Mech. Phys. Solids 36 (3) (1988) 251-283.

[36] A. Chrysochoos, Vers une reformulation des transition de phase du premier ordre, Revue Roumaine des Sciences Techniques, Mécanique Appliquée 43 (3), 1999.

[37] H. Louche, Analyse par thermographie infrarouge des effets dissipatifs de la localisation dans des aciers, Thesis of Montpellier University, 1999.

[38] A. Chrysochoos, H. Louche, Analyse thermographique des mécanismes de localisation dans des aciers doux, C.R. Acad. Sci. Paris, t. 326, Série II b, 1998, pp. 345-352.

[39] H. Louche, A. Chrysochoos, Analyse thermodynamique de la localisation de la déformation dans des aciers par thermographie infrarouge, Actes Photomécanique 98 (1998) 207-215.

[40] B. Wattrisse, M. Némoz-Gaillard, J.-M. Muracciole, A. Chrysochoos, etude cinématique des phénomènes de localisation dans un acier par intercorrélation d'images, Actes Photomécanique 98 (1998) 21-28.

[41] B Wattrisse, Etude cinématique de localisation dans les aciers par intercorrélation d'images de granularité, Thesis of Montpellier University, 1999. 